Draft VERSion June 17, 2021

Preprint typeset using $\mathrm{IAT}_{\mathrm{E}} \mathrm{X}$ style emulateapj v. 5/2/11

\title{
OB ASSOCIATIONS AT THE UPPER END OF THE MILKY WAY LUMINOSITY FUNCTION
}

\author{
Mubdi Rahman $^{1,2}$, Christopher D. Matzner ${ }^{1}$ \& Dae-Sik Moon ${ }^{1,3}$ \\ Draft version June 17, 2021
}

\begin{abstract}
The Milky Way's most luminous, young and massive $\left(\mathrm{M} \gtrsim 10^{4} \mathrm{M}_{\odot}\right)$ star clusters and $\mathrm{OB}$ associations have largely evaded detection despite knowledge of their surrounding H II regions. We search for these clusters and associations within the 40 star forming complexes from Rahman \& Murray in the 13 most luminous WMAP free-free emission sources of the Galaxy. Selecting for objects with the dust-reddened colors of OB stars, we identify new candidate associations using the 2MASS point source catalog. In 40 star forming complexes searched, 22 contain cluster/association candidates with sizes and masses in the range of $3^{\prime}-26^{\prime}$ and $10^{2.3}-10^{5} \mathrm{M}_{\odot}$. Of the 22 candidates, at least 7 have estimated masses $\gtrsim 10^{4}$ $\mathrm{M}_{\odot}$, doubling the number of such massive clusters known in the Galaxy. Applying our method to a statistically similar set of test locations, we estimate that $3.0 \pm 0.6$ of our 22 candidate associations are unrelated to the star forming complexes. In addition, the apparent extinctions of our candidate associations correlate well with the predictions from a Galactic model. These facts, along with the clear detection of a known OB association and the previous spectral verification of one cluster found by this method, validate our method. Only one of the searched WMAP sources remains without a candidate. In 8 of the most luminous WMAP sources, the candidate associations can account for the observed free-free flux. With our new compilation, the Galactic census of young, massive stellar associations may now be about half complete.
\end{abstract}

Subject headings: open clusters and associations — stars: massive — stars: formation — infrared: stars

\section{INTRODUCTION}

A spiral galaxy's metabolism is dominated by the effects of its most massive $\left(\mathrm{M} \gtrsim 10^{4} \mathrm{M}_{\odot}\right)$ young stellar clusters and $\mathrm{OB}$ associations. Not only are these clusters and $\mathrm{OB}$ associations the birthplaces of a substantial fraction of a galaxy's stars, but they also dominate the energetic feedback within the galactic disk and drive diskhalo interactions through the production of superbubbles (McKee \& Williams 1997; Chu 2008; Weaver et al. 1977; Norman \& Ikeuchi 1989; Reynolds et al. 2001). While the upper end of the cluster mass function (CMF) has been derived through Galactic H II region observations (McKee \& Williams 1997; Murray \& Rahman 2010), the powering clusters of the H II regions have mostly evaded detection. This is primarily due to the large distance and extinguishing column through the Galactic disk. Although a number of members of the upper end of the CMF have been identified and characterized, including NGC 3603 (Stolte et al. 2004) and the Galactic Center clusters (Figer et al. 1999), the census of the upper end is far from complete. This poses a problem for any global studies of the Galaxy's energy budget, star formation properties, turbulence, and overall ecology. Additionally, more complete knowledge of the upper end of the CMF is required for detailed comparison to other galaxies, critical to further understanding the role of star formation in galaxy evolution (Whitmore et al. 2007; Larsen 2009).

\footnotetext{
mubdi@pha.jhu.edu

${ }^{1}$ Department of Astronomy \& Astrophysics, University of Toronto, 50 St. George Street, Toronto, Ontario, M5S 3H8, Canada

${ }^{2}$ Department of Physics and Astronomy, Johns Hopkins University, Baltimore, MD 21218, USA

${ }^{3}$ Brain Pool Scholar, Korea Astronomy and Space Science Institute, Daejeon, Korea, 305-348
}

Recent work by Rahman \& Murray (2010, hereafter RM10) with data from the Wilkinson Microwave Anisotropy Probe (WMAP) and the Spitzer GLIMPSE survey has identified 40 star forming complexes (SFCs) within the Galaxy's most luminous free-free emission sources. Only one of the SFCs has a previously identified powering cluster (NGC 3603), indicating that the complexes may be harboring unknown luminous young massive clusters and OB associations. Notably, Rahman et al. (2011a) identified a candidate association for the most luminous SFC (the Dragonfish Nebula) based on the density of near-infrared (NIR) point sources, filtering non-member field stars using an extinction-based color-cut. The candidate association is located $9.7 \mathrm{kpc}$ through the Galactic disk with a line-of-sight extinction $1.0<\mathrm{A}_{K}<1.4 \mathrm{mag}$. Without the NIR color selection process, the association would remain hidden to visual or automated cluster search methods. Rahman et al. (2011b) confirmed this association with NIR spectra of a sample of its brightest members.

In this paper, we expand upon the method of Rahman et al. (2011a) to search for young massive clusters or OB associations in all of the SFCs identified in RM10. In $\S 2$, we discuss previous cluster searches. In $\S 3$, we outline the nature of the target SFCs. In $\S 4$, we discuss the use of the Two Micron All-Sky Survey (2MASS) for cluster searches and present the cluster search technique. In $\S 5$, we present our new candidate clusters and associations, discuss their statistical significance, and test the method through which they are identified. In $\S 6$, we estimate masses and luminosities for the candidates. In $\S 7$, we produce and discuss the most recent census of Galactic young massive clusters and OB associations. We summarize our conclusions in $\S 8$. 


\section{PREVIOUS CLUSTER SEARCHES}

Star clusters have historically been discovered in the visible wavelengths. However, these discoveries have been limited to the closest cluster populations due to the substantial dust obscuration through the disk. The advent of large NIR surveys has enabled the search for star clusters and OB associations despite this obscuration. Specifically, the 2MASS $(1.2-2.2 \mu \mathrm{m})$ and the Spitzer Galactic Legacy Infrared Mid-Plane Survey Extraordinaire (GLIMPSE, 3.6-8.0 $\mu \mathrm{m}$ ) have produced catalogs covering large areas of the Galactic plane in wavelengths where stellar flux dominates but extinction effects are minimized, although not completely eliminated (Skrutskie et al. 2006; Benjamin et al. 2003).

Dutra et al. (2003) and Bica et al. (2003) have systematically searched for star clusters in 2MASS, focused around optical and radio nebulae-primarily H II regions. Their search used visual identification of clustering on small images $\left(5^{\prime}-15^{\prime}\right.$ square) centered around the nebulae. Though this process, 346 new cluster candidates were identified. However, these efforts only searched for clusters around known nebulae, missing those not coincident with nebular emission (see $\S 3$ ). In a more automated manner, Froebrich et al. (2007) used the total on-sky stellar density of 2MASS point sources within $|b|<20^{\circ}$ to identify 1788 cluster candidates, of which 1021 are new. This method is less biased towards nebulacoincident clusters, but more susceptible to false positives. They estimated that their contamination rate of chance overdensities within their catalog is approximately $50 \%$. In both these cases, the searches are most sensitive to dense clusters, rather than diffuse regions. For instance, Froebrich et al. (2007) only detected a small clustered region within Cygnus OB2, rather than the entire $\mathrm{OB}$ association with a half-light radius of $13^{\prime}$, which is readily identifiable with 2MASS (Knödlseder 2000). Over $96 \%$ of the Froebrich et al. (2007) catalog candidates have a total central cluster density of greater than four sources arcminute ${ }^{-2}$, and over half greater than 10 sources arcminute ${ }^{-2}$. For the highly veiled case where only the most luminous stars are visible, typical source densities of $10^{4} \mathrm{M}_{\odot} \mathrm{OB}$ associations are expected to be $\sim 1.0(R / 10 \mathrm{pc})^{-2}(D / 10 \mathrm{kpc})^{0.84}$ sources arcminute ${ }^{-2}$, where $R$ and $D$ are the association radius and distance (Rahman et al. 2011a). For comparison, the typical stellar density of a 2MASS field in the Galactic Plane is between 14 and 17 sources arcminute ${ }^{-2}$ (\$4.1). Consequently, these previous 2MASS searches are insensitive to more dispersed OB associations. Similarly, Mercer et al. (2005) identified 92 new cluster candidates using similar automated and visual identification methods with the GLIMPSE point source catalog. All of the GLIMPSE cluster candidates have diameters of $3^{\prime}$ or less, indicating the same bias towards dense clusters. Since there are likely many more associations with diameters $>3^{\prime}$, such as Cygnus OB2, a systematic search optimized towards larger, more massive $\mathrm{OB}$ associations is required to find them.

For the candidate clusters identified in the works of Dutra et al. (2003), Bica et al. (2003), and Mercer et al. (2005), further photometric and spectroscopic studies have found high rates of false positive clusters (Borissova et al. 2005; Ivanov et al. 2005). Those clusters that are confirmed tend to have masses in the range of $10-1,000$ $\mathrm{M}_{\odot}$ and contain no stars with spectral types earlier than B, so cannot be significant ionization sources (Hanson \& Bubnick 2008; Soares et al. 2008). This suggests that most of the galaxy's largest ionizing clusters have not been identified.

The recent work by Bica \& Bonatto (2011) indicates that when candidate clusters are poorly populated or projected against dense stellar backgrounds, discerning a real cluster from a field density fluctuation (or asterism) is difficult or impossible. The effect poses a problem in the search for the most luminous clusters in the Galaxy located at significant distances; only the brightest members would be visible due to the substantial extinction and distances through the Galactic disk, even in the infrared regime. This creates the appearance of a poorly populated cluster. Further, most, if not all, of these clusters and associations should be located inside the Galactic disk, leading to a substantial confusing stellar background population. Therefore, these previous searches are best suited to finding nearby, compact clusters far from the Galactic mid-plane.

In summary, previous automated cluster searches are sensitive to compact clusters, like NGC 3603, but not to more diffuse associations like Cygnus OB2 and the Dragonfish. To thoroughly catalog the Galaxy's most luminous clusters using NIR surveys, one must exclude a large fraction of the field giants.

\section{WMAP-IDENTIFIED STAR FORMING COMPLEXES}

To identify the locations of the most luminous OB associations, we use the identification of their parental SFCs from the WMAP and Spitzer GLIMPSE survey (Murray \& Rahman 2010, RM10). The WMAP mission provides all-sky, high sensitivity $(\sim \mu \mathrm{K})$ microwave maps with five bands in the $23.5-90 \mathrm{GHz}$ range with a relatively large $\left(\sim 1^{\circ}\right)$ beam size. The five bands permit the separation of each of the Galactic "foreground" emission components; synchrotron emission, thermal dust emission, and free-free emission. The free-free emission almost entirely arises from reprocessed ionizing photons produced by the young, luminous (and massive) stars that are found en masse in the most luminous young clusters and OB associations. Murray \& Rahman (2010) identify free-free emission sources through the WMAP foreground map, finding that 14 sources are responsible for one-third of the total galactic ionizing luminosity. These regions are expected to be powered by young, very massive clusters or $\mathrm{OB}$ associations that contain sufficient O-type stars to produce the observed free-free emission. Within these 14 sources, only two have had previously identified powering clusters and associations, specifically the Galactic Center region and NGC 3603 (Murray \& Rahman 2010). Also notable is that many of the previously known massive OB associations, such as Carina and Cygnus OB2, are not enclosed by these 13 most luminous regions. This suggests the existence of ionizing clusters or associations more luminous than those previously identified.

The large $\left(\gtrsim 1^{\circ}\right)$ radii of the WMAP sources leads to significant confusion along the line of sight, especially in the sources towards the inner Galaxy. RM10 use the Spitzer GLIMPSE $8 \mu \mathrm{m}$ emission maps and radio recombination line radial velocities to separate the large WMAP sources into SFCs for the 13 most lumi- 
nous sources from Murray \& Rahman (2010), excluding the Galactic Center region. The observed SFCs differ in structure from the traditional model of a Strömgren sphere H II region: the complexes often appear as limbbrightened shells with the strongest nebular emission tracing large shells, typically $5^{\prime}$ to $30^{\prime}$ in radius, rather than in the central region. This gives the impression that a powerful central association has evacuated a zone of neutral gas, which it illuminates and ionizes. The brightest knots of emission in these shells, which often coincide with previously identified H II regions, could represent sites of triggered massive star formation. Further, Murray \& Rahman (2010) find that the majority of ionizing photons escape from the shells and produce diffuse ionization surrounding the entire region, often with radii $>1^{\circ}$, an effect previously inferred in the literature (Anantharamaiah 1985; McKee \& Williams 1997). While the classical Strömgren sphere interpretation of the SFC structure would suggest that the powering source should be located towards the center of the bright emission, this scenario suggests that the cluster is not expected to be coincident with the nebular emission for any but the youngest and smallest complexes.

Within the 13 investigated WMAP sources, RM10 identify 40 discrete SFCs with distances ranging from 2.5 to $15 \mathrm{kpc}$ and semimajor axes between $2^{\prime}$ and $67^{\prime}$. In some cases, the kinematic distance ambiguity is not resolved and two possible distances are identified. Since the WMAP sources are distant and projected against the crowded Galactic plane, we expect a great deal of confusion along the line of sight: while the inferred luminosities of these sources suggest that they contain massive clusters, they also likely contain a number of less-massive sources along the line of sight. Due to the large fraction of ionizing photons escaping the central area of the complex to radii similar to the distances between complexes, we cannot disentangle the different flux components from each of the sources. This makes it difficult a priori to determine which of the complexes are home to the massive clusters using ionization-based measures. In this work, we search for massive clusters and associations in all of the identified SFCs.

Previously, we have directed our attention to the most luminous of the SFCs, the Dragonfish Nebula. We developed a method using the 2MASS point source cata$\log$ to identify a candidate for the Galaxy's most luminous OB association, with a total stellar mass of $10^{5} \mathrm{M}_{\odot}$ (Rahman et al. 2011a). Follow-up NIR spectroscopy of bright stars within the candidate association confirmed the existence of Dragonfish Association with membership statistics consistent with the candidate's expected value (Rahman et al. 2011b). This example lends credence to the NIR color selection method as a means to identify candidate clusters. In this work, we use the method of Rahman et al. (2011a) to identify candidate clusters and OB association within the remaining SFCs from RM10.

\section{THE SELECTIVE STAR COUNT METHOD}

We refine the method of Rahman et al. (2011a) to identify candidate clusters and associations within the SFCs using the line-of-sight extinction through the Galactic plane. This filters non-cluster stars from candidate members, allowing the associations to be identified in areas of significant point source contamination. Schematically, the process is as follows: We isolate stars matching the NIR colors of O-type stars at graduated extinction levels. We then use the on-sky density of point sources matching the color cut to find overdensities consistent with the location of the SFC. Verifying from binned color-color and color-magnitude diagrams that the candidate cluster is not caused by a chance overdensity of projected Galactic structure based on the color distribution along the line of sight, we extract the candidate cluster's membership properties. We refer to this method as the Selective Star Count (SSC) method, which we describe in greater detail.

\subsection{The 2MASS Point Source Catalog}

To identify members of the candidate cluster, we use the 2MASS Point Source Catalog (Skrutskie et al. 2006). The survey covers the entire sky with $J(1.25 \mu \mathrm{m}), H$ $(1.65 \mu \mathrm{m})$, and $K_{S}(2.16 \mu \mathrm{m})$ bands, enabling consistent coverage of the SFCs in both the northern and southern skies. We limit our searches to the point sources with photometric quality flags of $\mathrm{A}, \mathrm{B}$, or $\mathrm{C}$, corresponding to a minimum signal-to-noise ratio of 5 for all of the $J, H$, and $K_{S}$ bands. The 2MASS catalogue is complete to $J \leq 15.8, H \leq 15.1$, and $K_{S} \leq 14.3$ magnitudes in the absence of confusion. However, the areas we investigate are highly confused causing the completeness limit to be up to 1 magnitude brighter. This prevents the detection of less luminous objects that are expected to be cluster members based on the stellar initial mass function (IMF).

The typical FWHM of the 2MASS point sources is 2.5 and quoted magnitudes are measured with apertures of $4^{\prime \prime}$, which will cause significant confusion for the most compact clusters or the dense areas of more diffuse OB associations (Skrutskie et al. 2006). Given the selected quality limit, these densely-packed confused sources will be excluded from our searches. Based on this constraint, we expect our search method to be insensitive to clusters with on-sky point source densities $\gtrsim 31$ arcminute $^{-2}$ due to overlapping apertures causing confusion. Within $1^{\circ}$ of the Galactic midplane, we find a total density of point sources in the target areas to be between 14 and 17 arcminute $^{-2}$ meeting the chosen quality cut. Therefore we expect such compact clusters to be readily identified by visual inspection of the images. In this sense, our SSC method is entirely complementary to search strategies of Bica et al. (2003), Dutra et al. (2003) and Mercer et al. (2005). We discuss this further in the case of NGC 3603 in $§ 5.1 .1$.

\subsection{Extinction-derived Color Selection}

The hypothesized powering clusters and associations of the SFCs should exist at large extinctions due to their distance through the Galactic disk. We make use of this to identify the cluster candidates on the basis that all cluster members should be similarly extinguished along the line of sight. Our method for uncovering candidate clusters is to identify overdensities of stars with colors consistent with massive stars at extinction ranges consistent with the distances to the SFC being probed.

All of the SFCs were identified using techniques sensitive to ionizing flux with large ionizing luminosities $\left(Q_{0} \gtrsim 10^{50.5} \mathrm{~s}^{-1}\right)$. The most luminous stars near the 
stellar upper mass limit have significantly smaller ionizing outputs $\left(Q_{0}=10^{49.6} \mathrm{~s}^{-1}\right.$ for an O3V star; Martins et al. 2005). The measured ionizing luminosities therefore require very large numbers of O-type stars to power them. For this reason we specifically search for overdensities of O-type stars with magnitudes and colors consistent with the distances and expected extinctions to the SFCs.

We use the NIR O-type star magnitudes and colors from Martins \& Plez (2006). We note that the colors of all O-type stars in $J-H$ and $H-K_{S}$ are constant, with $J-H=-0.11$ and $H-K_{S}=-0.10$, as expected, since these bands are in the Rayleigh-Jeans tail at the surface temperatures of O-type stars. These colors are, however, not unique to O-type stars; B- and early Atype stars are indistinguishable from O-type stars based on NIR colors alone to the accuracy of the 2MASS color measurements (Rahman et al. 2011a), and later stars are relatively more abundant; there is a roughly 8:1 ratio between O and B-type stars (Kroupa 2001). Only through their magnitudes can we make a distinction between $\mathrm{O}$ , B- and A-type stars, given a particular distance and extinction.

We take the intrinsic colors of O-type stars and redden them to arrive at our 2MASS color cuts. For the extinction law, we adopt that of Nishiyama et al. (2009), which gives the extinction ratios $\frac{A_{J}}{A_{K}}=2.89$ and $\frac{A_{H}}{A_{K}}=1.62$. We note that this is a departure from the canonical values for the extinction ratios due to a different power law slope for the relationship between extinction and wavelength $\left(A_{\lambda} \propto \lambda^{\alpha}\right)$; this determination uses a slope $\alpha=-2.0$, while the previously accepted value is $\alpha=-1.61$ (Cardelli et al. 1989). We adopt the Nishiyama relationship over the canonical as it is empirically determined from a more highly extinguished sample of stars that are more likely to be consistent with the extinctions that we probe in this work. The shallower slope has been found to be erroneous by Stead \& Hoare (2009), who use an even steeper power law slope of $\alpha=-2.14$.

The steeper slope of the extinction law changes the relationship between the $\mathrm{NIR}(>1 \mu \mathrm{m})$ and visual $(\sim$ $0.5 \mu \mathrm{m})$; rather than a ratio of $A_{V} / A_{K} \sim 10$, the new ratio becomes $A_{V} / A_{K} \sim 16$ (Nishiyama et al. 2008). However, the difference in the extinction ratios between the different laws in the NIR $(1-2.5 \mu \mathrm{m})$ bands is less than $20 \%$, which is inconsequential in the candidate cluster identification since we use only the NIR bands and the large color cuts used for the selection procedure.

We use the following relations for $A_{K}^{\prime}$, the estimated $\mathrm{O}$-star extinction in the K-band:

$$
\begin{aligned}
A_{K}^{\prime}(J-H) & =0.797(J-H)+0.0877 \\
A_{K}^{\prime}\left(H-K_{S}\right) & =1.66\left(H-K_{S}\right)+0.166
\end{aligned}
$$

We use these relations to select point sources consistent with O-type stars in a particular extinction range.

\subsection{The Nearest Neighbour Algorithm}

We analyze the density structure of 2MASS point sources using a nearest neighbour method, as described by Casertano \& Hut (1985). This method samples each of the points within the chosen color cut, providing an adaptive resolution, sampling the areas with more point sources more heavily than those areas without point sources. The primary advantage of this method over a simple spatial density map is that the resolution is adaptive; areas with large numbers of point sources will contain detail of density structures at a higher resolution than areas with a sparsity of sources. From each of the points, we measure the distance $r_{j}$ to the $j$-th nearest neighbour. We determine the local surface density, $\mu_{j}$, with an unbiased estimator:

$$
\mu_{j}=\frac{j-1}{\pi r_{j}^{2}}
$$

with $j>3$. From Casertano \& Hut (1985), the standard deviation of the estimator is:

$$
\sigma_{j}=\frac{\mu_{j}}{\sqrt{j-2}}
$$

To ensure the consistent visual appearance of the point source density variations regardless of the number of sources in the field, the specific value of $j$ to be used is a function of the total number of point sources in the field. If a constant $j$ value was used for all fields, those with large numbers of point sources would sample only very local variations of the surface density, while those with few point sources would smooth over all but the largest density structures in the field. The on-sky point source density varies based on the location in the Galactic plane, the color-cut chosen, and the width of the color-cut. The typical on-sky densities of the point sources can vary by an order of magnitude based on different color cuts since fewer stars with very red colors are cataloged in 2MASS than bluer stars. Consequently, using a constant $j$ value over all color cuts would dramatically change the scale of structure highlighted. So for each cut, we determine an optimal value for $j$ given a angular resolution based on the average number of point sources within a field:

$$
j=\left\lfloor\frac{\theta_{c r}^{2} N_{*}}{\Theta^{2}}\right\rfloor
$$

where $N_{*}$ and $\Theta$ are the total number of stars in and angular radius of the color-selected 2MASS field, and $\theta_{c r}$ is the characteristic resolution, corresponding to the mean resolution across the selected field. For the purpose of this investigation, we choose a characteristic resolution $\theta_{c r}=3.5^{\prime}$ based on the resolution used to identify the Dragonfish Association from Rahman et al. (2011a). At this characteristic resolution, typical $j$ values range from 15 to 30 for most regions with extinction cuts of $\Delta \mathrm{A}_{K}=0.1$. The densities are gridded onto an oversampled $\left(d<11^{\prime \prime}\right)$, uniformly-spaced grid using a Delaunay triangulation method from the Matplotlib python package (Hunter 2007) to simplify the analysis.

\subsection{Candidate Identification}

For each of the SFCs, we examine the surrounding $2^{\circ}$ field with 0.1 steps in the $\mathrm{A}_{K}^{\prime}$ color cut in the range of $0.0<\mathrm{A}_{K}^{\prime}<2.0$. We enlarge the surrounding field to $3^{\circ}$ for SFCs with semi-major axes larger than $30^{\prime}$. In each extinction step, we produce the nearest neighbour onsky density map, and visually identify cluster candidates coincident with the on-sky position of the SFC position and the $8 \mu \mathrm{m}$ morphology from RM10.

Using small steps in the color cut allows for effective filtering of field stars from candidate clusters: while the 
typical point source density of unfiltered sources ranges from 14 to 17 sources arcminute ${ }^{-2}$ (§4.1), the typical field densities within the color cuts range from $0.05-0.5$ sources arcminute ${ }^{-2}$. The substantial decrease in the field source density due to the color filtering enables the detection of more diffuse clustering than has been possible in previous searches $(\S 2)$.

Our adoption of sequential extinction cuts over the entire range allows for the filtering of effects due to the structure of the Galaxy itself. For instance, a significant effect of Galactic structure is the appearance of patchy but mostly latitude-dependent stratification of the source density above and below the Galactic plane caused by the limited thickness of the extinction-producing gas disk and also by the appearance of all thick disk and halo stars at a particular color range (Figure 1). These large scale effects can be visually separated from localized overdensities with ease.

Once a candidate is identified, the extinction range is refined to enhance the prominence of the overdensity, and a final density map is produced. From this map we visually identify the candidate's geometric boundaries, including its centroid position, semi-major and semi-minor axes. Once the boundaries are chosen, we determine the total number of enclosed sources, $N_{T O T}$. To constrain field contamination, we measure the mean $\left(N_{B G}\right)$ and standard deviation $\left(\sigma_{B G}\right)$ of the number of field stars surrounding the candidate in regions with the same area as the identified candidate.

To better distinguish between real clusters and asterisms, we analyze each candidate cluster with binned color-color and color-magnitude diagrams as in Rahman et al. (2011a). Both sets of diagrams allow us to distinguish between a true overdensity of sources (evidence for the existence of a cluster or association) or a shift of sources from one color to another (evidence of a local extinction feature). From these diagnostics, we find that none of the overdensities appear to be shifts of points in color-color or color-magnitude space, which is additional evidence against the alternative hypothesis of a local extinction feature.

\section{CANDIDATE CLUSTERS/ASSOCIATIONS}

In Table 1, we present the candidate clusters/associations identified within the SFCs. For SFCs with multiple candidates, an alphabetical key is used to distinguish between them. To quantitatively measure the statistical significance of the extracted clusters as compared to the natural field variations, we define the extraction significance:

$$
\mathrm{ES}=\frac{N_{T O T}-N_{B G}}{\sigma_{B G}}
$$

with $N_{T O T}, N_{B G}$, and $\sigma_{B G}$ as defined in $\S 4.4$.

Two of the candidate associations (those within SFC 8 and 30) have extraction significance values of less than unity; these candidates are identified solely based on their morphology as compared to the SFC and have significant large scale structure in the field surrounding them that produces large background variations.

The candidates have characteristic radii between $3^{\prime}$ and $20^{\prime}$, with a median of $\sim 7^{\prime}$. This is consistent with the sensitivity of the SSC method to larger diffuse structures than in previous cluster searches (see §4.4). Accordingly, these candidates would not have been identified in the previous searches. While the SSC method is more sensitive to diffuse structures, the ellipses are chosen to contain the most prominent areas of the overdensity and do not necessarily represent the outer boundaries of the clusters. We expect that K-giants are the primary contaminants of all the new candidates, since they tend to be the dominant population of visible red stars in the 2MASS survey (Robin et al. 2003).

The candidate boundaries are visually chosen to encompass the most centrally-condensed, smooth portions of the overdensity, avoiding the lower isodensity contours, which are more flocculent, when possible. This corresponds to an extraction contour of $1-\sigma$ to $2.8-\sigma$ above the local background variation level. We investigate any bias in the visual identification of candidates, the chosen extraction contour, and their effects on their extracted parameters with a suite of tests described in Section 5.3.

We present the stellar density diagrams and the $8 \mu \mathrm{m}$ images from the Spitzer GLIMPSE or Midcourse Space Experiment (MSX) in Figures 2 to 8 . These images indicate the SFC location from RM10 and the candidate cluster location.

Many of the candidates are not centered on the nebulosity visible in the $8 \mu \mathrm{m}$ images. This may be an indication of the age of the cluster; if the cluster is young, it would not have had sufficient time to evacuate its surrounding material. Consequently, it would appear at the same location as the $8 \mu \mathrm{m}$ emission. If the cluster is older, it would have evacuated its surrounding material and likely blown a bubble. A cluster in this stage would appear separate from the material it is illuminating. In addition, the SFCs identified by RM10 are visually identified and may not correctly reflect the location or the size of the SFC based on the limited data. If confirmed, the candidate clusters will enable the refinement of the SFC parameters.

Since the candidate extinctions were determined independently from the position and distance to their host SFC, we can use the modeled extinction from Marshall et al. (2006) as a consistency check. We present the comparison of the candidate cluster extinction ranges with the modeled extinction in Figure 9. To determine the model extinctions, we use the SFC kinematic distance form RM10 in Table 1 . We also indicate the possible model extinctions of SFCs without a kinematic distance ambiguity solution and the location of the Dragonfish association from Rahman et al. (2011a). We find the correlation coefficient of the two extinctions to be 0.75 when using the more consistent distance in the cases of the distance ambiguity. The correlation is not simply the result of choosing the most consistent distance, however. We have randomly shuffled the inferred and modeled values of $A_{\mathrm{K}}$ five thousand times, choosing, for each shuffling, the most consistent model extinction. The results are shown in Figure 10: without shuffling, the ratio of modeled to observed extinction is $1.1 \pm 0.22$ dex (standard deviation in the logarithm); with shuffling, the ratio becomes $0.9 \pm 0.36$ dex. The increased scatter in the shuffled sample strongly supports the validity of the candidate clusters, at least on a statistical basis. This correlation provides strong evidence for the reality of the candidate clusters. For sources with a distance ambigu- 

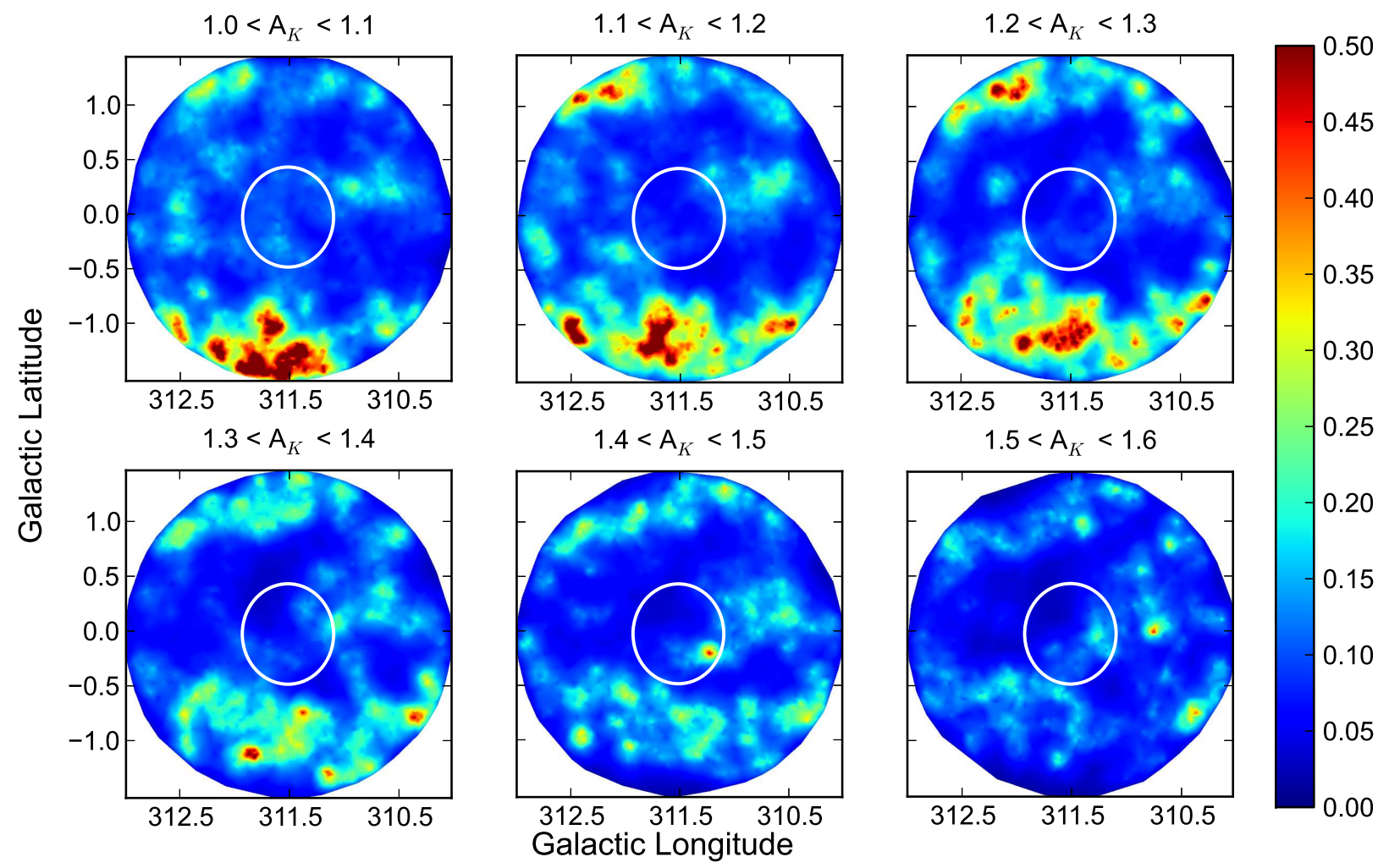

Figure 1. 2MASS Point Source Density Maps at the location of SFC 31 (white circle) with extinction cuts between $\mathrm{A}_{K}=1.0$ and 1.6 (left to right, top to bottom). The color bar indicates the point source density in sources arcminute ${ }^{-2}$. Galactic structure effects produce the long horizontal streak-like features that appear in the first extinction cut symmetrically $1^{\circ}$ above and below the galactic plane and narrow in subsequent cuts. These features are visually filtered from the selection process. Despite the appearance of compact overdensities, these cuts do not contain any identified candidate clusters, since they are likely higher-density pockets associated with the Galactic structure features. This can be compared to the candidate identified in this region at a lower extinction cut (Table 1; Figure 6).

ity, the modeled extinction provides support in favour of one of the two possibilities. We find this correlation to be strikingly better than the correlations between extinction and distance.

\subsection{Previously Identified Clusters}

An important test of the success and the sensitivity of the SSC method is identifying known massive clusters and associations within 2MASS. For this purpose, we use the cases of NGC 3603, the powering cluster of SFC 28, and Cygnus OB2. We discuss these two cases below.

\subsection{1. $N G C 3603$}

A notable cluster that was not detected by the SSC method is the massive cluster within NGC 3603 that powers SFC 28. This very compact cluster is mostly contained within $33^{\prime \prime}$, requiring high spatial resolution to discern individual members (Stolte et al. 2004). At the resolution of $2 \mathrm{MASS}$, the central cluster is highly confused, as can be seen in Figure 11. This prevents individual sources from being identified, so they are missing from the 2MASS PSC. In fact, only 17 point sources are identified in the central region that pass the desired quality cut for the SSC method (inset of figure 11). Further filtering point sources to those with colors corresponding to O-type stars at the extinction of NGC 3603, we are restricted to 12 point sources. Although these 12 sources do represent a local maximum in the color-selected source density within the cluster's boundary, this is neither significant when compared with the background variation, nor representative of the cluster's true stellar population. In redder color cuts, this region is seen as a void in point source density. This is consistent with our expectation that the SSC method is insensitive to compact clusters, such as those that can be easily identified visually.

Despite this, the SSC method is useful to investigate the entire area of the NGC3603 natal cloud for a diffuse population surrounding the dense cluster. We suspect that these are the candidate clusters we identify in SFC 28 , on the edge of the region. They appear at a significantly higher extinction than NGC $3603\left(\Delta \mathrm{A}_{K} \sim 0.4\right)$. If they are not unrelated regions farther across the Galactic plane, they could be regions deeply embedded within the cloud, consistent with the CO morphology from Grabelsky et al. (1988). In either case, these candidates are not the powering source of SFC 28.

\subsubsection{Cygnus OB2}

To ensure that the SSC method can identify known OB associations, we use Cygnus OB2 as a test case. This association, located in the outer Galaxy, has a substantially reduced background stellar population in comparison to 



Figure 2. Spitzer GLIMPSE $8 \mu \mathrm{m}$ image (left) and the on-sky density diagrams (right) of the 2MASS point sources. The source and extinction ranges are indicated above each figure. The green solid ellipse indicates the location of the SFC from RM10, while the red dashed ellipses indicate the location of the candidate clusters. The color bar indicates the point source density in sources per square arcminute. 

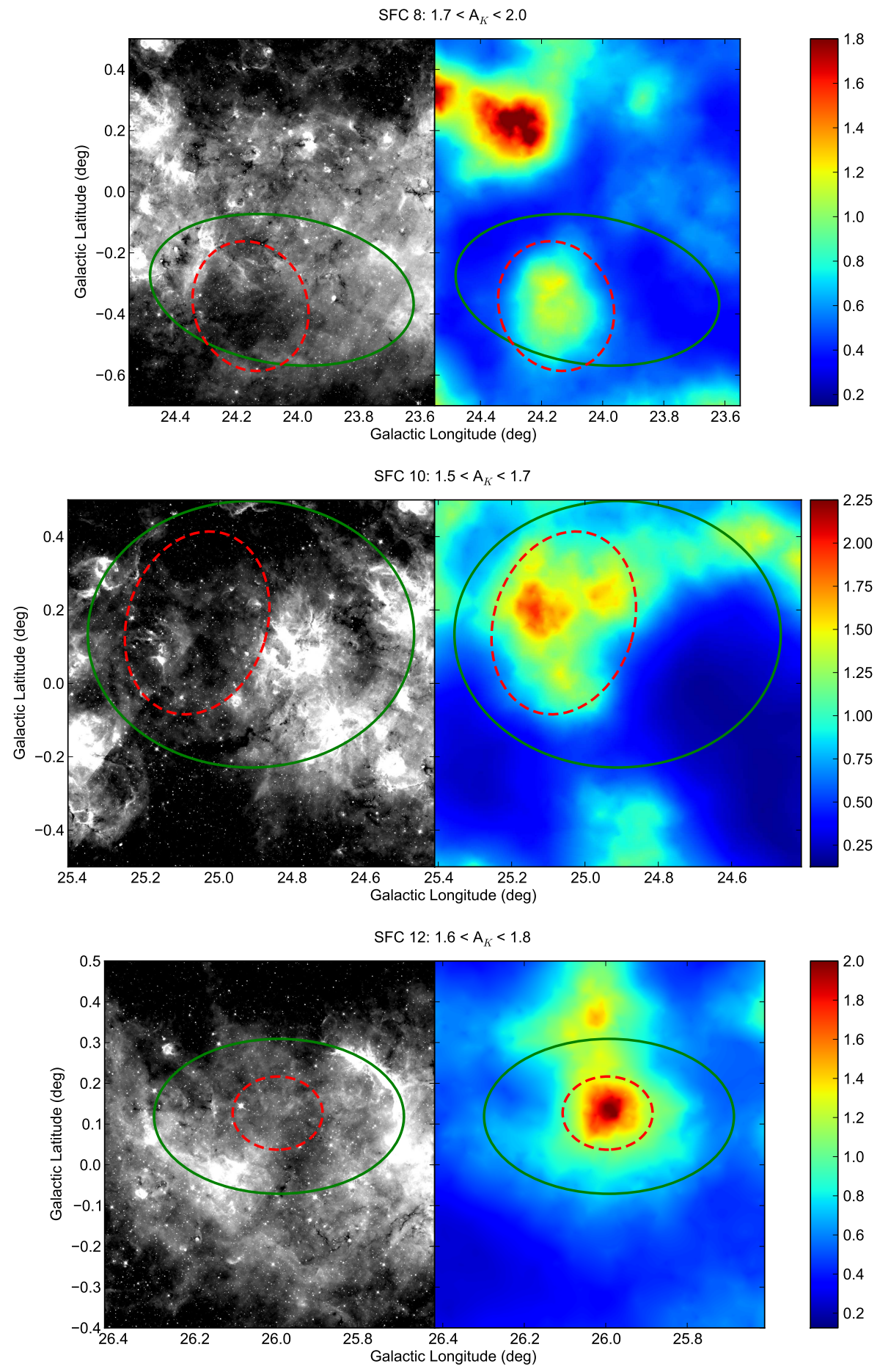

Figure 3. Spitzer GLIMPSE $8 \mu \mathrm{m}$ image (left) and the on-sky density diagrams (right) of the 2MASS point sources. The source and extinction ranges are indicated above each figure. The color bar and annotations are the same as Figure 2. 

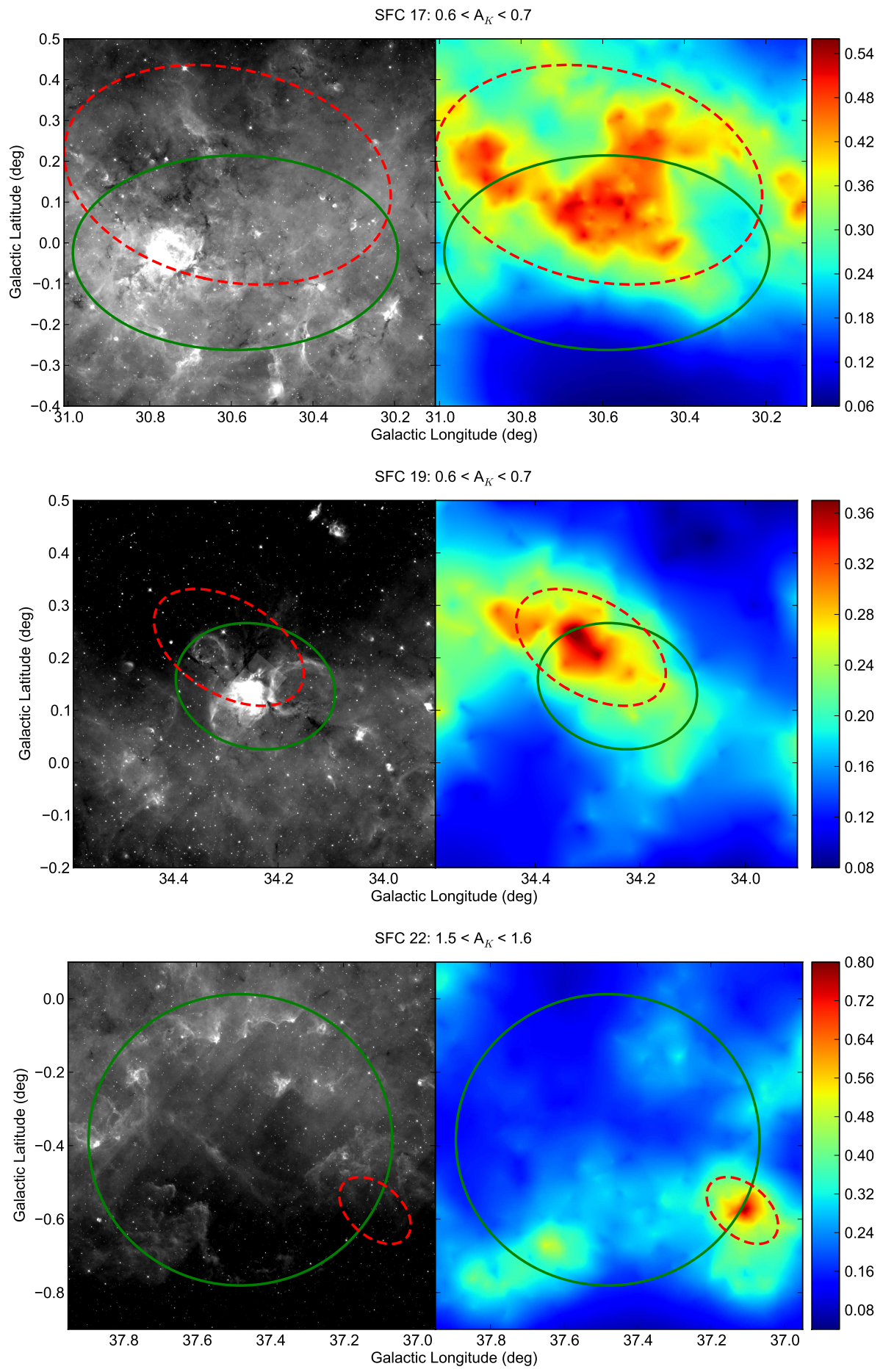

Figure 4. Spitzer GLIMPSE $8 \mu \mathrm{m}$ image (left) and the on-sky density diagrams (right) of the 2MASS point sources. The source and extinction ranges are indicated above each figure. The color bar and annotations are the same as Figure 2. 

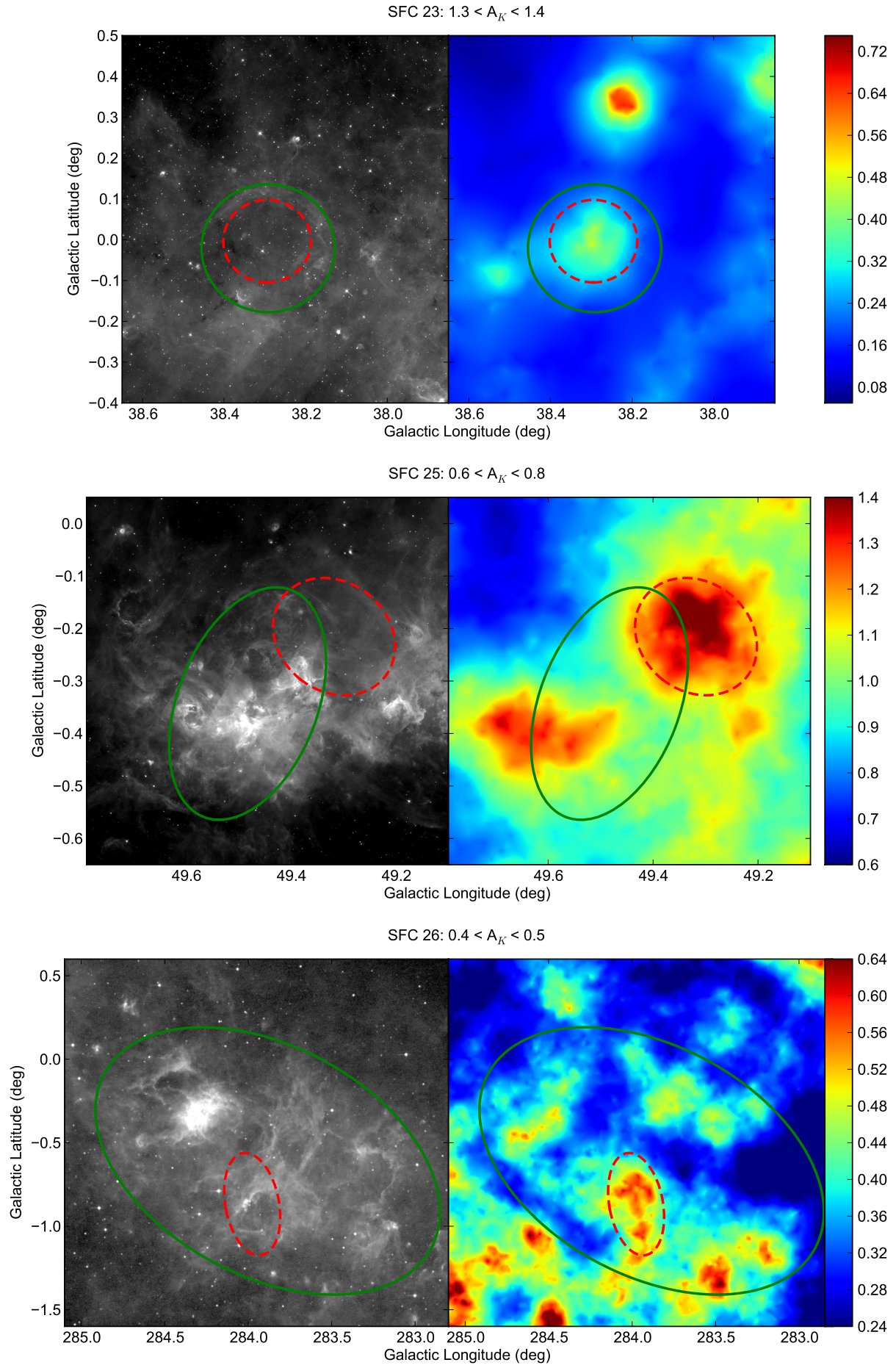

Figure 5. Spitzer GLIMPSE $8 \mu \mathrm{m}$ image (left) and the on-sky density diagrams (right) of the 2MASS point sources. The source and extinction ranges are indicated above each figure. The color bar and annotations are the same as Figure 2 . We use the MSX $8 \mu \mathrm{m}$ image for SFC 26 due to Spitzer GLIMPSE coverage limits. 

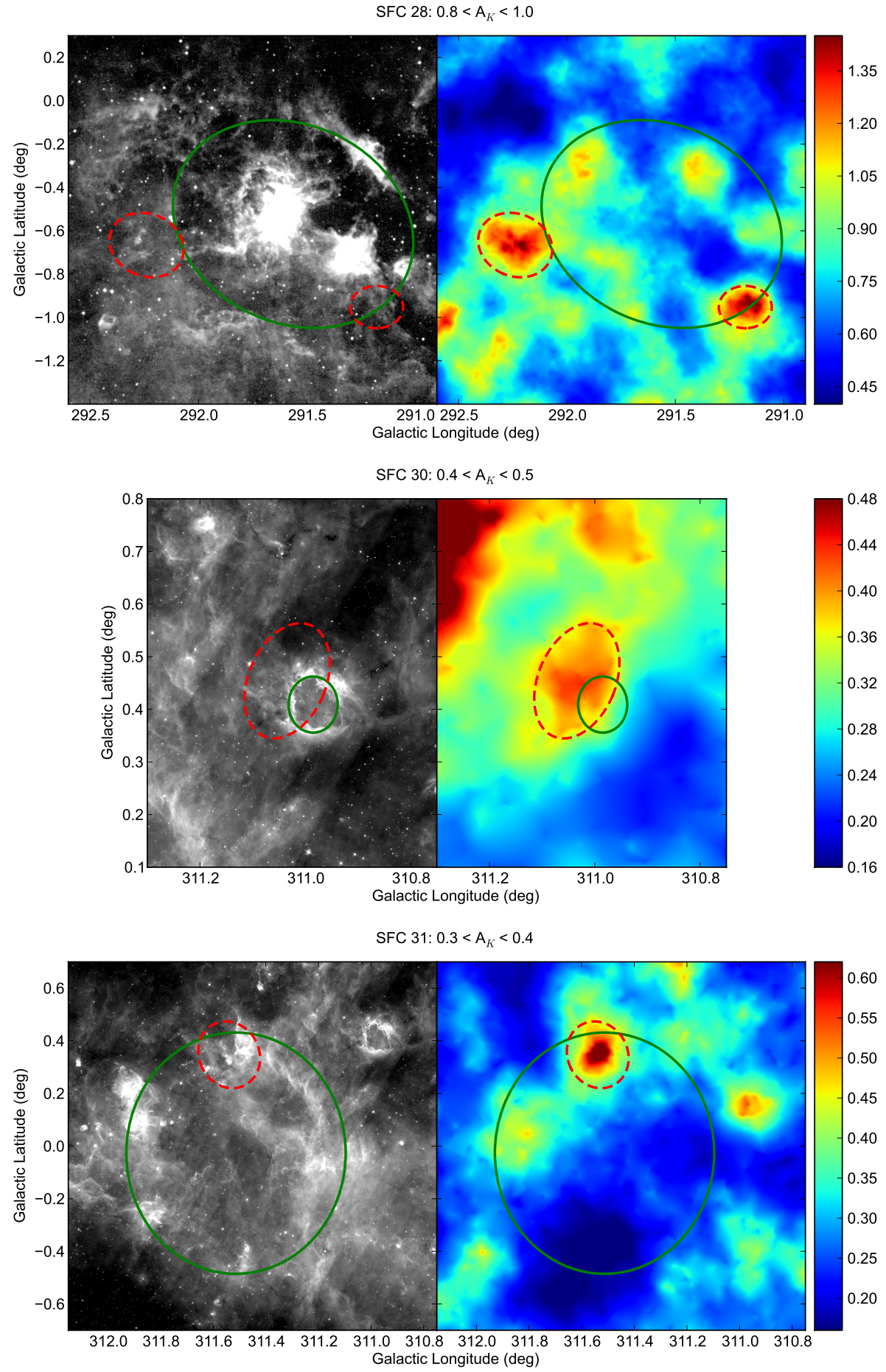

Figure 6. Spitzer GLIMPSE $8 \mu \mathrm{m}$ image (left) and the on-sky density diagrams (right) of the 2MASS point sources. The source and extinction ranges are indicated above each figure. The color bar and annotations are the same as Figure 2 . We use the MSX $8 \mu \mathrm{m}$ image for SFC 28 due to Spitzer GLIMPSE coverage limits. 

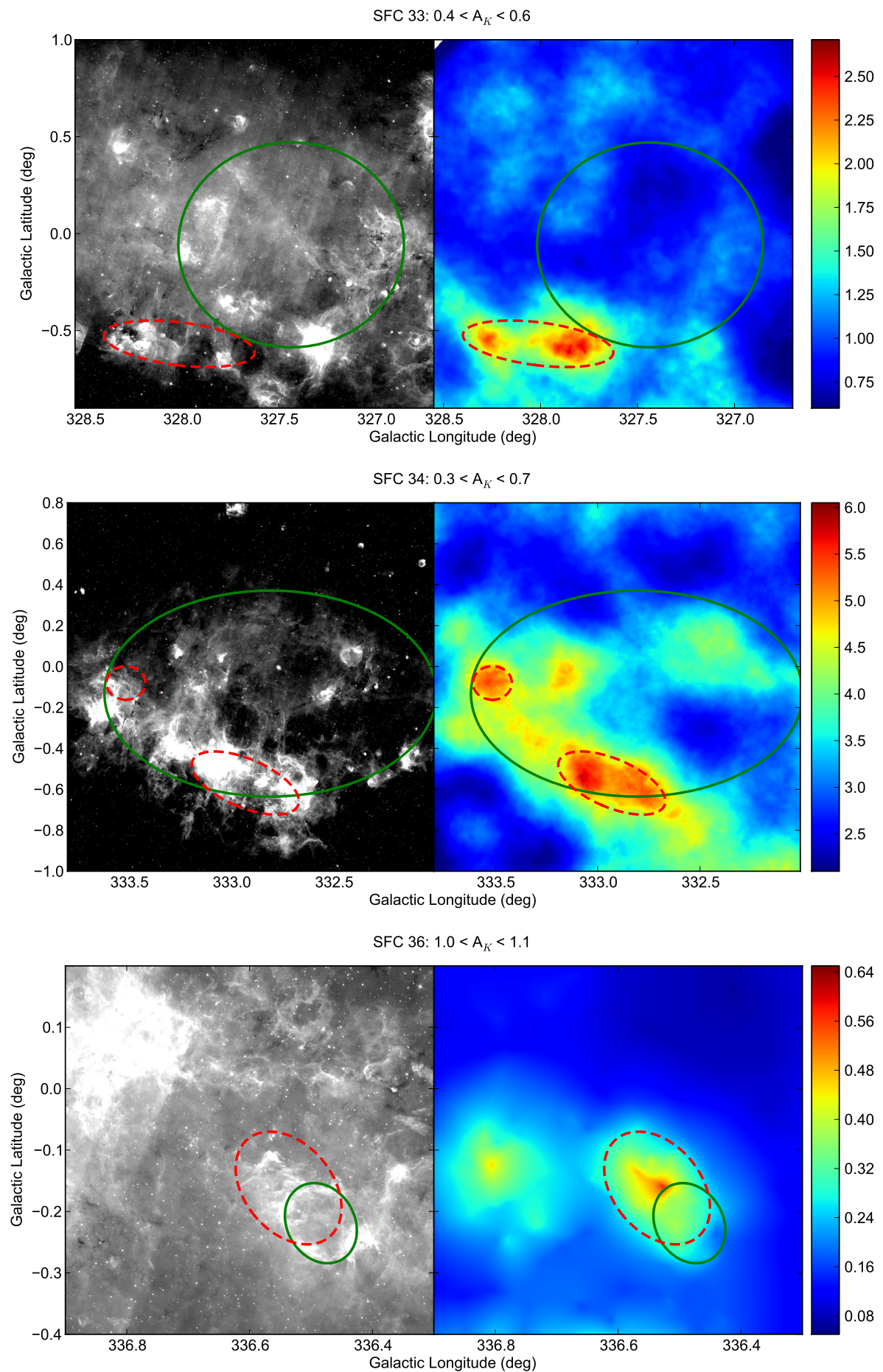

Figure 7. Spitzer GLIMPSE $8 \mu \mathrm{m}$ image (left) and the on-sky density diagrams (right) of the 2MASS point sources. The source and extinction ranges are indicated above each figure. The color bar and annotations are the same as Figure 2. 

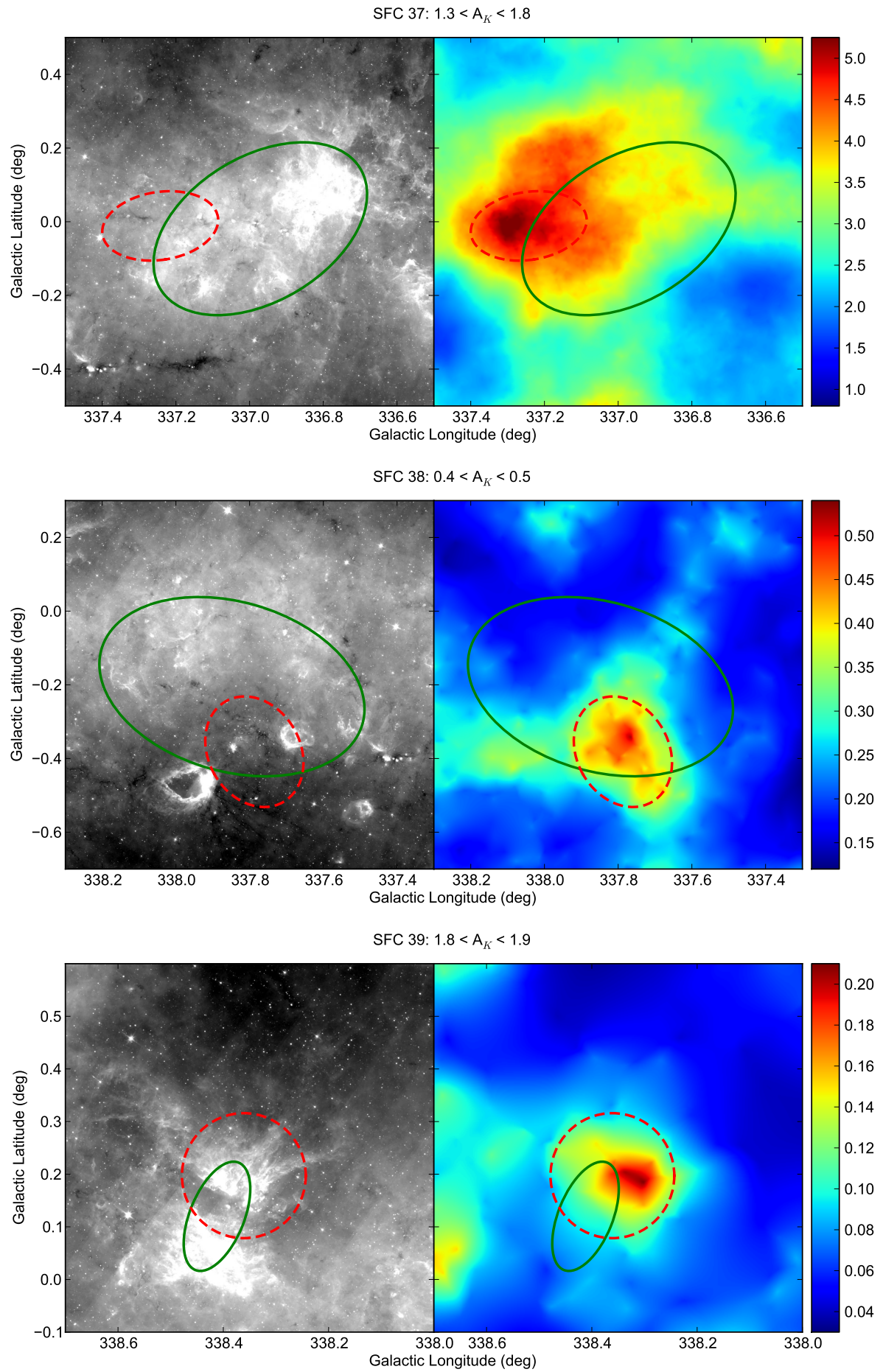

Figure 8. Spitzer GLIMPSE $8 \mu \mathrm{m}$ image (left) and the on-sky density diagrams (right) of the 2MASS point sources. The source and extinction ranges are indicated above each figure. The color bar and annotations are the same as Figure 2. 

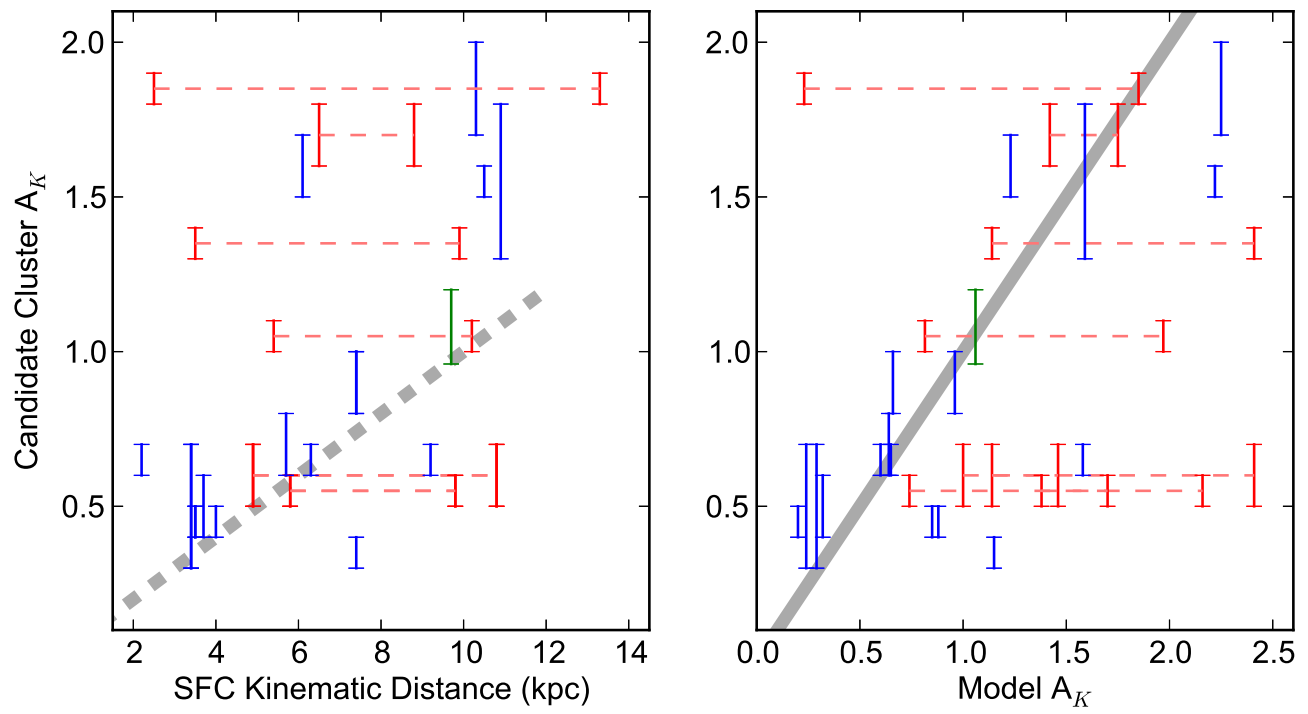

Figure 9. A comparison between the extinction range of all identified candidate clusters to the kinematic distance of their host SFC from RM10 (left) and with the model extinction from Marshall et al. (2006, right). The diagonal is indicated with the gray line on the right, and the local distance to extinction relationship $\left(A_{K} / D=0.1 \mathrm{mag} \mathrm{kpc}^{-1}\right)$ is indicated with the dashed gray line on the left. This relation breaks down for distances greater than $6 \mathrm{kpc}$, as compared to the relation between the candidate and model extinction that remains tight for all candidate distances. The connected red ranges indicate the SFCs without a kinematic distance ambiguity resolution. The green range indicates the location of the Dragonfish Association properties from Rahman et al. (2011a). As no information about the kinematic distance was used in identifying the candidates, the correlation between the candidate and model extinction is not caused by a bias in the SSC method.

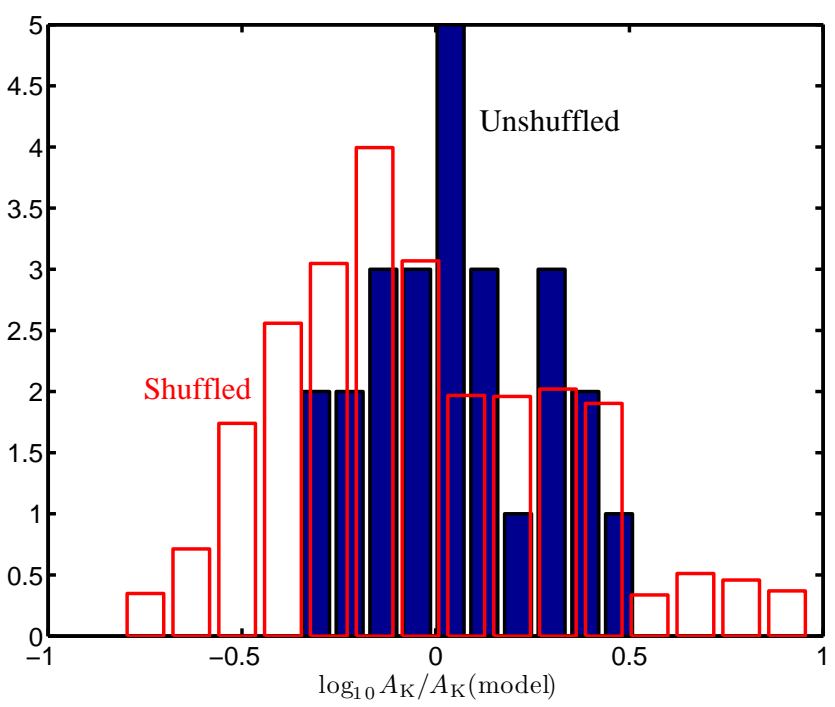

Figure 10. A histogram of the ratios of the candidate cluster extinction to the modeled extinction from Marshall et al. (2006) in the filled histogram. The outlined histogram is the result of shuffling the candidate cluster extinctions 5000 times, normalized to the number of candidates in our sample. The larger scatter of the shuffled histogram provides additional support for the reality of the candidates. 


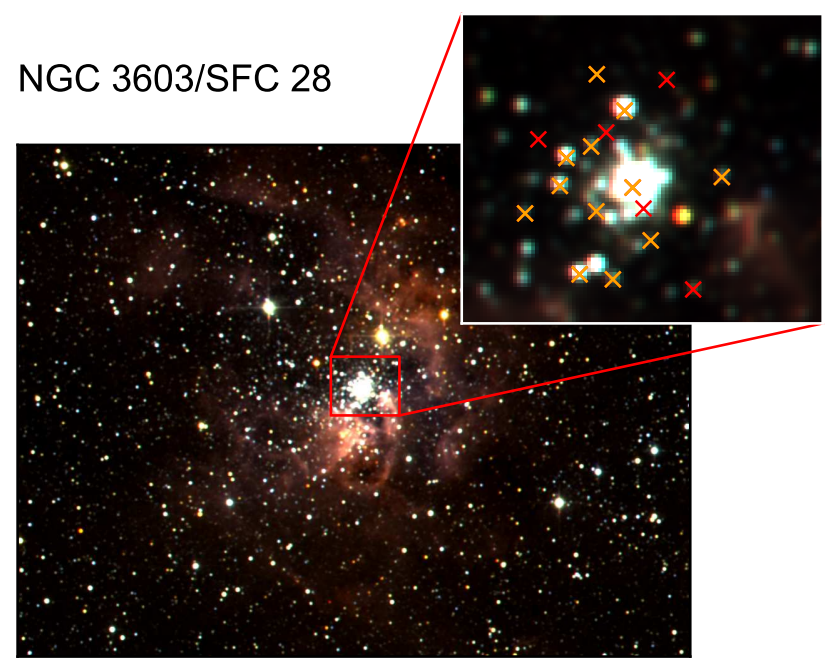

Figure 11. NGC 3603 region from 2MASS in J (blue), H (green) and K (red) with the central cluster magnified (inset). The crosses indicate the locations of the only 2 MASS point sources that pass the required quality cut within $33^{\prime \prime}$ of the cluster center. The orange crosses are the point sources that meet the color cut of the cluster, while those in red are the sources that do not.

the clusters in the inner galaxy. Therefore, the association can be identified using total stellar density without extinction cuts, as has been done by Knödlseder (2000).

Using our method, we easily identify Cygnus OB2 (Figure 12) at an extinction range of $0.5<\mathrm{A}_{K}<0.8$, with position, structure and extinction consistent with the values determined by Knödlseder (2000). The typical background level in the field is $0.3-0.6$ sources arcminute ${ }^{-2}$, approximately $10 \%-20 \%$ of the peak density of the association. If we choose the boundary of the cluster to be the isodensity contour at 1.2 per arcminute $^{-2}$, the average rate of line-of-sight contaminating sources within the association boundaries is $<0.28$, allowing for high-yield extraction of association members from the field.

\subsection{Statistical Significance}

In $\S 5.1$, we have discussed the success and sensitivity of the SSC method using previously identified clusters and associations. However, the problem that has plagued previous cluster candidate identification methods has been the overwhelming number of false positives. We conduct a test to determine the rate of false positive candidate identifications through the SSC method.

We generate a list of 40 artificial SFCs with positions, dimensions and position angles randomly chosen to match the distribution of actual SFCs on the sky: the artificial locations are generated from a uniform random distribution between $270^{\circ}<l<90^{\circ}$ and $-1^{\circ}<b<1^{\circ}$. The position angles, semimajor axes and the axial ratios were generated by producing linearly interpolated cumulative distribution functions from the real SFC list. Using the SSC method, we attempt to identify "candidate clusters" for these artificial SFCs, with the same criteria as in $§ 4.4$. This test was conducted five times. From the five lists of artificial SFCs, the rate of identified candidates is $3.0 \pm 0.6$ sources, which is a much smaller yield than the 22 actual SFCs with identified candidates. It should be noted that the artificial candidates may be actual clusters or associations related to less luminous WMAP sources on the sky from Murray \& Rahman (2010).

In addition to the false positive test, the correlation between the modelled extinction and the candidate extinction ( $\S 5$; Figure 9) provides strong support for the reality of the clusters identified by the SSC method. Finally, the spectroscopic confirmation of the Dragonfish Association (Rahman et al. 2011b) provides additional, circumstantial evidence of the clusters identified by this method. These independent lines of evidence indicate that most $(\sim 86 \%)$ of our cluster candidates are indeed associated with their host SFCs, and are their dominant engines of ionization and dynamical power.

\subsection{Testing Cluster Extraction}

We conduct a pair of tests to determine any bias in the extracted parameters of the candidate clusters. First, we test the accuracy of the number of extracted sources as a function of extraction radius. We select a $2 \mathrm{MASS}$ field centered on $(l, b)=\left(343.11^{\circ},-0.50^{\circ}\right)$ with the extinction-based color cut $0.6<A_{K}^{\prime}<0.7$ and insert randomly generated clusters. The background level of sources in this field is $0.24 \pm 0.11$ arcminute $^{-2}$. The clusters were generated with 40 visible stars in a $7^{\prime}$ radius, corresponding to a "true" extraction significance of $\sim 3$ for the entire radius of the cluster in this field and a central peak point source density of 0.8 sources arcminute $^{-2}$. The worst case scenario for a poor extraction yield is a shallow density profile; this leads to the highest number of stars at large radii that may be missed with the selection of a smaller extraction radius. To test this worst case scenario, we model the cluster with a uniform stellar density in three dimensions. We insert 1000 randomly generated clusters into different random locations in the field. We extract each of the clusters with extraction radii ranging from $0.5^{\prime}$ to $20^{\prime}$.

In Figure 13, we present the results of this simulation. The $1-\sigma$ extraction boundary corresponds to an extrac- 


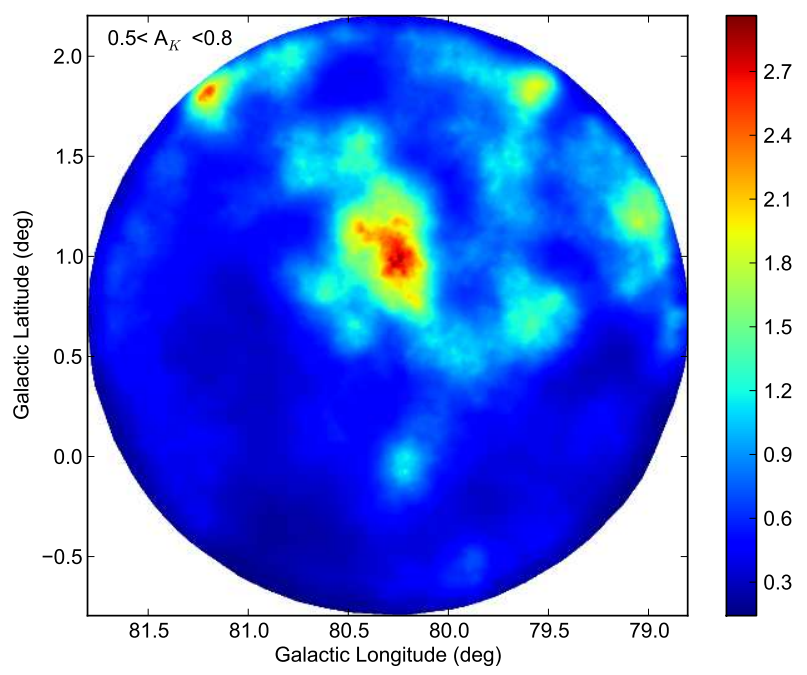

Figure 12. The on-sky density diagram of 2 MASS point sources between $0.5<\mathrm{A}_{K}<0.8$ in the Cygnus OB2 region. The color bar indicates the point source density in sources arcminute ${ }^{-2}$

tion radius that is $\sim 0.8$ times the size of the model cluster radius, and extracted cluster source numbers that are $\sim 80 \%$ of the inserted numbers. Arbitrarily larger extraction radii produce extracted cluster source numbers that approach $100 \%$ of the inserted numbers, but with growing error at larger radii. When a more conservative $2-\sigma$ or $3-\sigma$ extraction radius is chosen, the extracted star numbers are $50 \%$ and $30 \%$ of the inserted cluster numbers, underestimating the total cluster population given this worst-case radial density model. This indicates that the chosen selection boundaries are either correctly estimating or underestimating the number of stars extracted.

Next, we conduct a test of the visual cluster identification and extraction method to determine what biases exist in the selection of the candidate clusters. For this test, we generate artificial clusters with a random distribution of positions, number of sources, and density profiles, place them in a real 2MASS field, and extract their positions, sizes, and number of sources with background estimation in the same manner as with the actual candidate clusters.

We generate 200 artificial clusters containing between 29 and 67 sources. We fix the truncation radius of the clusters at $7^{\prime}$, the median of the semimajor axes from the candidate clusters. We vary the $2 \mathrm{D}$-density profile of clusters using a Sérsic probability distribution of the form:

$$
P(R) \propto \exp \left(-2\left(R / R_{T}\right)^{1 / n_{s}}\right)
$$

where $R_{T}$ is the truncation radius, and $n_{s}$ is the Sérsic index. Larger Sérsic indices correspond to more centrally concentrated distributions, while smaller indices produce more shallow profiles. For simplicity in comparison, we choose discrete integer values between 1 and 4 for the Sérsic indices.

We choose the same $3^{\circ}$ MASS field $\left(l, b=343.11^{\circ}\right.$, $\left.-0.50^{\circ}\right)$, and extinction-based color cut $\left(0.6<A_{K}^{\prime}<\right.$ $0.7)$ from the earlier test. This field is free of identified candidate clusters, but does contain the stratified Galactic structure feature similar to that shown in Figure 1.
We represent the number of artificial cluster sources inserted as $N_{\text {input }}$, and the number of field stars at that location as $N_{b g}$. The number of sources estimated in the cluster after background subtraction using the visual extraction method is represented as $N_{s u b}$.

Of the 200 artificial clusters inserted, 160 were successfully identified and extracted. The centroiding of the identified clusters is quite accurate; the mean difference between the inserted and extracted central positions of the identified test clusters is $1.4 \pm 0.9$ arcminutes and only 7 identified clusters have positional differences greater than $3^{\prime}$. The 40 unidentified test clusters are typically confused with Galactic structure and the size distribution is skewed towards clusters with smaller numbers of points. In addition, the unidentified test clusters are skewed towards larger Sérsic indices. This indicates that the visual selection method is biased against smaller clusters and those with peakier density profiles. This is consistent with the expectation that we are not sensitive to particularly compact clusters.

From the 160 artificial clusters extracted, we can determine the accuracy of retrieving the initial cluster population. We find that $70 \%$ of clusters have a fraction of extracted sources to the inserted sources, $f_{\text {ext }}=$ $N_{\text {sub }} / N_{\text {input }}$, between 0.6 and 1.0 with a mean value of 0.84 (Figure 14 inset). The typical measured ES values of the artificial candidates range from 2.5 to 5.5. We find no strong correlations between $n_{s}, N_{\text {input }}$ or $N_{b g}$ with $f_{\text {ext }}$. However, we find that there is an inverse correlation of the ratio of $N_{\text {input }}$ and $N_{b g}$ with $f_{\text {ext }}$ (Figure 14); in the small number of cases where we overestimate the size of the cluster (by up to a factor of two), these clusters are a small fraction of the background level of the region $\left(N_{\text {input }} / N_{b g}<0.35\right)$. Overall, these tests indicate that the method is robust in recovering the original cluster population and position, with a tendency to underestimate the total population size.

\section{MASS \& LUMINOSITY ESTIMATES}




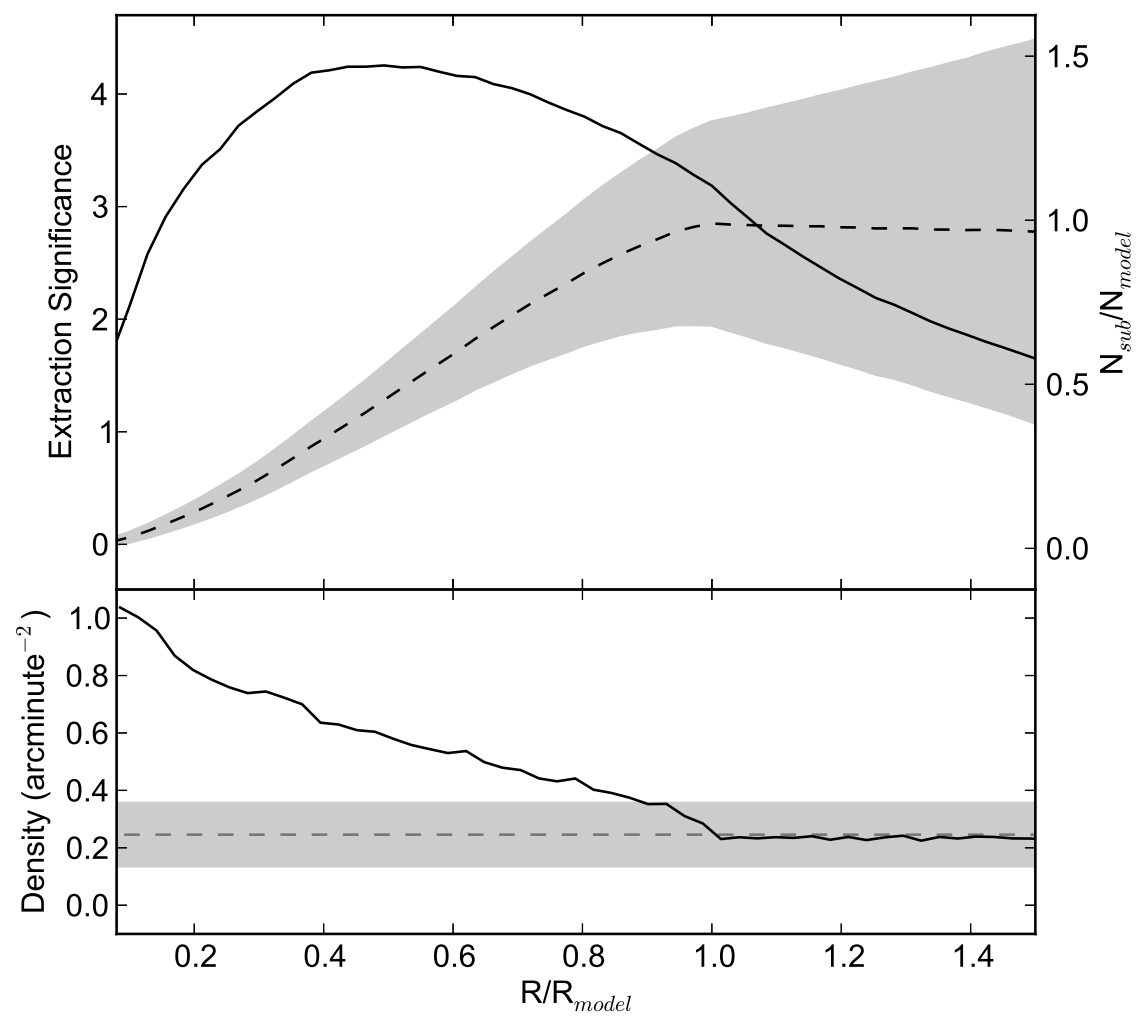

Figure 13. The results of the model cluster extraction simulation. The mean extraction significance (solid line) and mean fraction of model stars extracted (dashed line) from all 1000 insertions are plotted in the top frame. The shaded area indicates the error in the fraction extracted based on the background variation. The mean point source surface density of all insertions is plotted in the bottom frame (solid line), with the background density level $\left(0.24\right.$ arcminute $^{-2}$; dashed line) and $1-\sigma$ background variation $\left(0.11\right.$ arcminute arc $^{-2}$; shaded region) indicated. The extraction radius $(\mathrm{R})$ is normalized to the radius of the inserted modeled cluster $\left(\mathrm{R}_{\text {model }}=7^{\prime}\right)$.

We estimate the physical properties of the candidate clusters to assess if the identified candidates can produce the observed free-free flux of their parent WMAP source (Table 2). We note that the stated boundaries from the candidate identification and extraction method are only visually identified based on a clear overdensity from the local background variation ( 55 ). Consequently, the extracted clusters will likely exclude the diffuse outskirts of the clusters or associations, especially when the background level of contaminating sources is particularly high. Such diffuse populations can produce significant contributions to the total complex population, as has been recently observed in the Carina Nebula where previously undiscovered OB stars were identified in Xray observations, doubling the massive stellar population (Povich et al. 2011). Although we found in the previous section that our method is likely to underestimate clusters' true population, we adopt a conservative approach and do not attempt to correct for the extraction efficiency.

We estimate the least massive stars that are extracted in the candidate clusters by using the observed 2MASS $K_{S}$-band magnitude limit from $\S 4.1$, with the mean extinction range of the candidate and the kinematic distance of the SFC from RM10. In cases where the kinematic distance is ambiguous, we use the distance most consistent with the Marshall extinction (see Figure 9). We use the absolute magnitude tabulations from Martins \& Plez (2006) for O-type stars and from Pickles (1998) for all other stars. We also use Martins et al. (2005) for the O-type star mass determinations and Andersen (1991) for all other stars. From the mass of the least massive star and the number of stars extracted in the candidate cluster $\left(N_{t o t}-N_{B G}\right.$ in Table 1$)$, we determine a total cluster mass $\left(M_{*}\right)$ and ionizing luminosity $\left(Q_{0}\right)$ integrating over an IMF. To maintain consistency with previous work, we use the modified version of the Muench et al. (2002) IMF from Murray \& Rahman (2010). The mass of the candidate was estimated by scaling the Muench IMF to the number of stars down to the least massive star extracted, and then integrating over the mass function to determine the total mass. The ionizing luminosity was determined by using the mass-to-ionizing luminosity relationship from Murray \& Rahman (2010). Note that we do not make any correction for the multiplicity of sources.

Finally, we determine the expected free-free flux contribution of the candidate at $\nu=90 \mathrm{GHz}$. We also visually classify the candidates based on their location with respect to the identified SFC and the $8 \mu \mathrm{m}$ morphology. We indicate that they are either "centrally-located" if they appear towards the center of a shell-like structure 


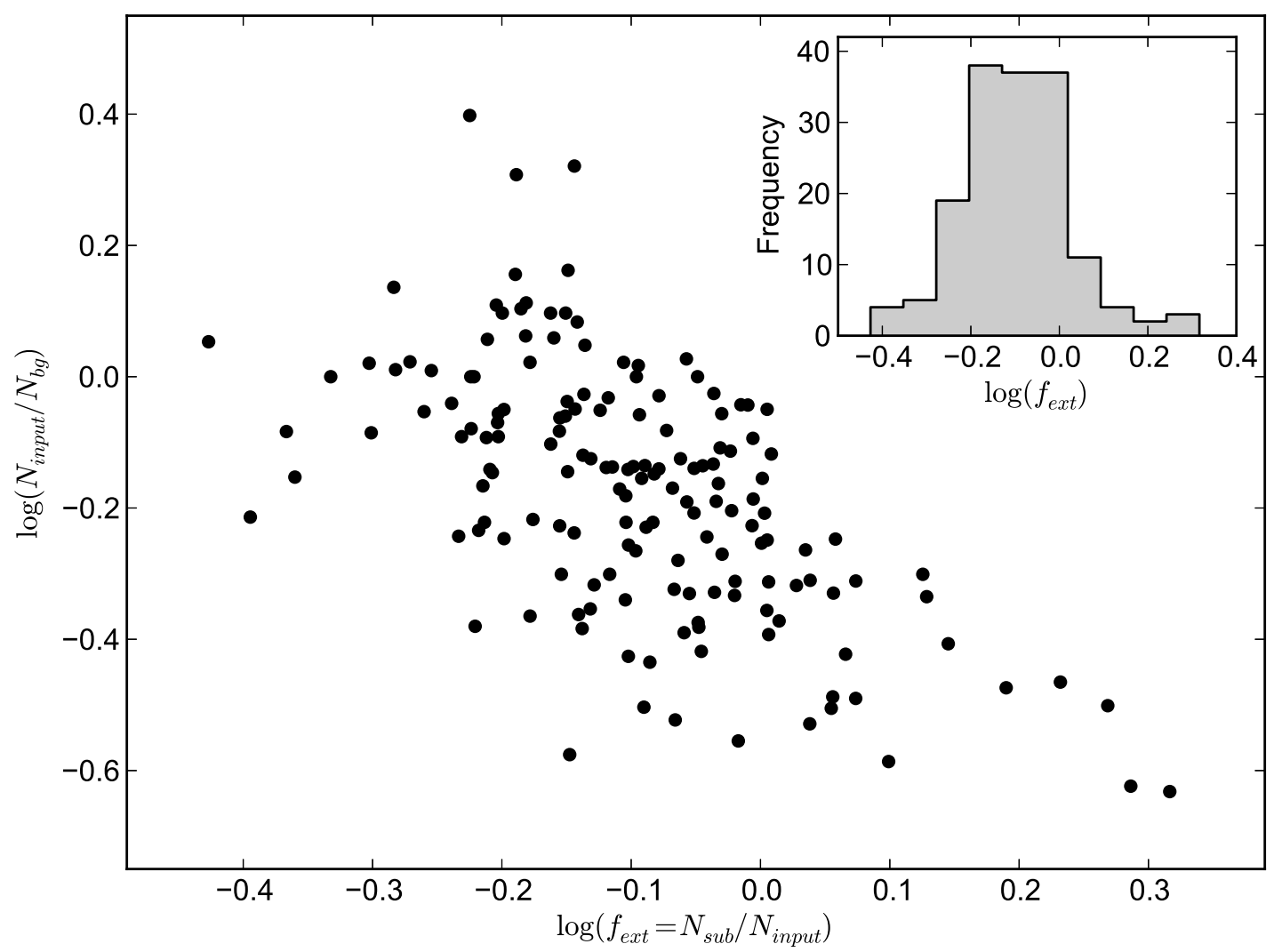

Figure 14. The results of the cluster extraction test using artificial clusters in a Galactic 2MASS field centered on $(l, b)=\left(343.11^{\circ}\right.$, $\left.-0.5^{\circ}\right)$. The ratio of the extracted point sources to the artificially inserted point sources, $f_{\text {ext }}$, vs. the ratio of the inserted point sources to the number of field sources in the background. We present the histogram of $f_{\text {ext }}$ of test clusters in the inset. The visual extraction method generally estimates a cluster population of between $60 \%$ to $100 \%$ of the inserted cluster population. The method only estimates a larger number of points than inserted into the cluster when the cluster is small in comparison to the number of background sources.

or the entire SFC, or "shell-located" if they appear on the edge of a shell or SFC.

In Table $1, N_{s u b}$ is the number of sources in the candidate cluster once the background level is subtracted $\left(N_{T O T}-N_{B G}\right)$. We include the Dragonfish Association properties from Rahman et al. (2011a). For the WMAP sources with multiple identified candidate clusters, we include totals for the cluster masses, ionizing luminosities and free-free emission fluxes.

We note that the latest stellar type of all extracted sources is between O- to A-type stars. All of these hot stars will have similar Rayleigh-Jeans colors in the NIR. Therefore, we do not expect cluster members to be excluded based on having colors that fall outside of the color cut.

The total cluster masses of the candidates range from 150 to $10^{5} \mathrm{M}_{\odot}$; perhaps not surprisingly, some appear to be low-mass objects unrelated to their host regions. In these cases, the estimated ionizing luminosity and freefree emission flux are overestimated as the IMF is poorly sampled on the upper end and the mass-to-ionizing luminosity conversion breaks down. This has been discussed in detail by Kennicutt et al. (1989). Therefore the freefree flux contribution from these clusters will be much smaller than those tabulated since the mass-to-ionizing luminosity relation is unsuitable for a poorly sampled IMF. By ionizing luminosities of $\log \mathrm{Q}_{0} \sim 50$ or cluster masses greater than $1600 \mathrm{M}_{\odot},>10$ O-type stars are required to ionize the complex and the IMF should be sufficiently well sampled to allow the accurate use of the mass-to-ionizing luminosity relationship (Martins \& Plez 2006).

We find the sum of the estimated free-free emission fluxes to be roughly consistent with the WMAP measurement for G34, G37, G298, G327, and G337. For G30 and G49, the identified candidates appear to be centrally located, but the smaller-than-expected mass, ionizing luminosity, and free-free flux most likely come from a diffuse component of the cluster/association. Should these candidates be confirmed, an in-depth investigation, possibly in the X-rays, will be required to identify the extended or diffuse population. For G283 and G327, only shell-located regions are identified. In the case of G327, the lack of sharp shell structure in the bubble in $8 \mu \mathrm{m}$ and 2MASS stellar overdensity structure (see Figure 7, top panel) may indicate that the original driving cluster of the bubble has evolved beyond its ionizing luminous stage, and the ionizing population of the complex is a more diffuse, triggered population. In source G291, we identify two possible triggered clusters; however the dom- 
inant cluster in the region, NGC3603, has been previously identified and was not detected by the SSC method (see $§ 5.1 .1)$.

In some cases, such as G332, multiple candidates are identified at the same (or reasonably close) distances and extinction ranges. These candidates are likely associated and may represent more widespread star formation across a molecular cloud or complex. Accordingly, the total embedded cluster mass in these situations would be the sum of the individual "clustered" regions, plus the diffuse component that we do not recover from the extraction process.

The total expected free-free emission luminosities of the candidates in G24 and G332 substantially exceed the measured flux from WMAP. There are at least six possibilities to account for this: (1) one or more of the candidates within the WMAP source are false positives, (2) the extraction has overestimated the number of member stars by a substantial fraction, (3) the kinematic distance to the SFC is closer than the actual distance, (4) the cluster is older than 3.9 Myr, the age at which the most massive stars have left the main sequence (Bressan et al. 1993), (5) a higher-than-average fraction of the ionizing photons are absorbed by dust, and (6) an unusually large fraction of the ionizing photons escape the region, or escape the Galaxy. To determine whether it is case (1) or (2), follow-up astrometric or spectral confirmation of the cluster is required. In case (3), the distances to the ionizing sources can be unambiguously determined though trigonometric parallax, if strong maser sources associated with the $\mathrm{H}$ II regions are available. In case (4), the evolutionary state of the candidate requires accurate determination of the spectral type of candidate sources to determine the earliest stars in the cluster and their individual evolutionary state. If this is the case, these clusters may harbor young core-collapse remnants of very massive stars, such as black holes or magnetars.

With these caveats, we find candidates to central powering clusters that can account for the observed WMAP free-free emission flux within G24, G30, G34, G37, G49, G298, G332, and G337. Of the original WMAP sources investigated in RM10, only G10 does not have any candidate clusters identified through this method, either centrally located or on the shell.

\section{COMPLETING THE UPPER END OF THE GALACTIC CLUSTER MASS FUNCTION}

Our knowledge of the upper end of the Galactic cluster mass function (CMF) has long been recognized as incomplete. This has been established through comparison to galaxies with similar masses and star formation rates as the Milky Way (Larsen \& Richtler 2000; Larsen 2009), and comparing the known cluster/association population with the inferred H II mass function (McKee \& Williams 1997; Hanson \& Popescu 2008). These comparisons show that up to $90 \%$ of young, massive clusters and OB associations are yet to be found.

Table 3 presents the current census of known massive, young $(\lesssim 4 \mathrm{Myr})$ clusters and $\mathrm{OB}$ associations in the Galaxy. This census includes all known clusters and associations with masses similar to or greater than $10^{4} \mathrm{M}_{\odot}$, in addition to the massive candidates from this work. The age limit of 4 Myrs ensures that even the most massive stars, which produce the bulk of the ionizing luminosity, are still on (or near) the main sequence (Murray \& Rahman 2010). This effect is prominently seen in the case of Westerlund 1, where despite the large cluster mass $\left(>10^{4.7} \mathrm{M}_{\odot}\right)$ and rich population of Wolf-Rayet and late-O type stars (Brandner et al. 2008), the cluster is not detected as a prominent free-free emission source in WMAP (Murray \& Rahman 2010). If the candidates from this work are confirmed with the minimum masses estimated, the census of young, massive clusters and OB associations is doubled with 7 new candidates.

Notably, the age restriction of the census excludes the red supergiant clusters recently discovered with ages $>10$ Myrs and masses $>10^{4} \mathrm{M}_{\odot}$ (Figer et al. 2006; Davies et al. 2007; Clark et al. 2009). The massive stellar component of these clusters has entirely evolved off the main sequence, and consequently the clusters have an insignificant ionizing luminosity. This is corroborated by the lack of any reprocessed free-free emission towards the clusters from the WMAP free-free emission maps (Murray \& Rahman 2010).

\subsection{Estimates of Completeness}

The completeness of the census can be estimated through CMFs from previous work for other galaxies in the visible wavebands, such as the Antennae and M83 (Fall et al. 2009; Chandar et al. 2010). Ivanov et al. (2010) estimate that the Milky Way contains $\geq 81 \pm 21$ clusters with masses of $10^{4}-10^{5} \mathrm{M}_{\odot}$ and ages up to $\sim 25 \mathrm{Myr}$, assuming all clusters within $6 \mathrm{kpc}$ are known (Piskunov et al. 2008). This estimate can be scaled to our 4 Myr age limit using the age distribution from Chandar et al. (2010, $\frac{d N}{d \tau} \propto \tau^{-1}$ ) with a minimum age of 1.3 Myr based on the youngest clusters observed. The scaled estimate is 31 clusters in the Milky Way, of which Larsen (2009) would predict that 8 are bound. This is consistent with the estimate made by McKee \& Williams (1997), who estimate the number of OB associations with $\mathrm{M}>10^{4} \mathrm{M}_{\odot}\left(\right.$ or $\left.\mathrm{Q}_{0}>10^{50.8} \mathrm{~s}^{-1}\right)$ to be 29 .

Another independent measure of completeness is a comparison to the H II region luminosity functions of other galaxies. We compare to the H II region luminosity functions from Kennicutt et al. (1989), using a minimum $\mathrm{H} \alpha$ luminosity of $L(H \alpha)>10^{38.6} \mathrm{erg} \mathrm{s}^{-1}$ which is similar to the $10^{4} \mathrm{M}_{\odot}$ cutoff. In the most analogous cases to the Milky Way in terms of morphological class, NGC 6384 (an Sb galaxy) contains 38 sufficiently luminous H II regions, while NGC 7741 (an SBc galaxy) contains 17. Smith \& Kennicutt (1989) show that the H II luminosity function of the Milky Way is comparable to those of $\mathrm{Sb}-\mathrm{c}$ galaxies, indicating that using the $\mathrm{Sb}-\mathrm{c}$ galaxies as a guide is a valid estimation of the total young, massive clusters in the Galaxy.

Both of these estimates of the total number of massive $\left(>10^{4} \mathrm{M}_{\odot}\right)$ clusters/associations in the Galaxy are comparable to 15 , the number of associations in our census, of which seven are presented here (Table 3). Since the estimates of the number of young, massive clusters expected is between 17-38, we infer from this that our knowledge of young, massive clusters in the Milky Way is now about half complete.

\subsection{Remaining Galactic Plane Targets}


This work is based on the luminosity-selected sample of SFCs from RM10 on the basis that their ionizing luminosities are from young, massive clusters hosted by the SFCs. However, this work was not a comprehensive search through the entire Galactic plane. We can determine the likely regions of young, massive clusters that remain hidden by comparing our census to the catalog of giant H II regions from Conti \& Crowther (2004). As noted in RM10, previously identified individual regions closely packed together are likely associated in larger complexes (see $\$ 2.1$ in RM10 for a detailed discussion). For the purpose of this comparison, we group the giant H II regions from Conti \& Crowther (2004) into 27 degree-sized zones in the Galactic plane. Each Galactic plane zone consists of 1-7 giant $\mathrm{H}$ II regions.

Of the 27 Galactic plane zones, young massive $\left(\gtrsim 10^{4}\right.$ $\left.\mathrm{M}_{\odot}\right)$ clusters or associations have not been identified in 18. Within the 18 zones without young massive clusters, 6 have previously known powering clusters with masses less than $10^{4} \mathrm{M}_{\odot}$, and 5 have candidates identified in this work also less than $10^{4} \mathrm{M}_{\odot}$. We list the zones lacking a young, massive cluster in Table 4, tabulating the Galactic Plane Region (in Galactic Longitude), their kinematic distance(s), and their names where applicable. For the 5 zones with candidates identified in this work, they have lower mass limits $>10^{3.2} \mathrm{M}_{\odot}$. Because our search is not sensitive to the diffuse outskirts of clusters/associations, it is possible that the candidates in the 5 zones have masses larger than $10^{4} \mathrm{M}_{\odot}$. Further observations and analysis are required to better constrain the total masses of these clusters.

The advent of new Galactic plane NIR surveys, such as the UKIRT Infrared Deep Sky Survey (UKIDSS; Lawrence et al. 2007) and the Vista Variables in the Via Lactea Survey (VVV; Minniti et al. 2010), will enable more sensitive probes for clustering than has been possible with 2MASS. Both UKIDSS and VVV are significantly deeper than 2MASS ( $\sim 4$ magnitudes in the $\mathrm{K}$ band), with a much smaller point source function $(\sim 4$ times smaller). Consequently, applying the SSC method in these surveys would enable the detection of more highly extinguished and less massive clusters, since the greater sensitivity allows for more highly reddened and intrinsically less luminous stars to be detected. In addition, these surveys would better probe more compact clusters, where the 2MASS point source function size has rendered them confused. However, two challenges will arise in using deeper surveys; because they are more sensitive to the less luminous members of a cluster, the assumption of Rayleigh-Jeans NIR colors will break down and a more complex search strategy following the expected color of the extinguished main-sequence will be required. Secondly, the increase in sensitivity will also increase the number of field sources; to better separate field and cluster sources, the explicit use of the luminosity information will be required. However, the potential in these new surveys is not limited to finding the remaining Galactic massive cluster and association population, but also better characterizing the candidate clusters and associations detected in this work.

We note that this work searches for candidate clusters with extinctions of $A_{K}<2.0$, while the extinction model from $\S 5$ indicates that significant portions of the Galactic plane will have extinctions of greater than that.
This includes a number of candidates from this work if you accept the far kinematic distance from the SFC. The currently used 2MASS data is not sufficiently populated with highly-reddened stars to allow the SSC work due at high extinctions. However, the deeper NIR surveys will allow searches for more highly extinguished clusters and associations.

Twelve of the 27 Galactic plane zones have not been probed in this work, leaving a substantial discovery space for the SSC method to be applied. Recently, Lee et al. (2012) have identified SFCs in the remaining WMAP free-free sources not originally covered in RM10, which provides a new sample of morphologically-identified regions to probe. Investigating this remaining SFCs may enable the completion of the entire upper end of the Galactic CMF.

\section{CONCLUSIONS}

We have used the 2MASS point-source catalog to search for massive clusters and association candidates within the SFCs from WMAP, looking for the brightest stars, taking into account the line of sight extinction. Including the previous work of Rahman et al. (2011a), we find that 22 of the 40 SFCs host a candidate cluster that has been identified through our method, either central to the SFC or on its periphery. The extracted candidates have semimajor axes of $3^{\prime}-26^{\prime}$. The average extraction significance of the candidates is 2.4 . The candidates with smaller extraction significances are selected based primarily on morphology.

We discuss the case of two previously identified clusters/associations: NGC 3603, which is unidentified through our method due to the compact nature of the cluster, and Cygnus OB2, which we readily identify and whose properties we accurately constrain. We find the false positive rate to be $3.0 \pm 0.6$ out of 22 identified. We test the visual identification method and find it to be robust in determining the population of the cluster. The strong correlation between the candidate extinctions and the modelled extinction based on the SFC distances provides strong support for the reality of the clusters. This is reinforced by the spectroscopic confirmation of the Dragonfish Association, originally identified the same method of Rahman et al. (2011b).

We estimate the masses and luminosities of the candidates, showing that the candidates can account for the observed WMAP free-free flux for most of the investigated sources. Of the WMAP sources investigated, only one (G10) lacks any candidate clusters or associations. Finally, we provide a Galactic census of the young, massive clusters and $\mathrm{OB}$ associations. With the newly identified candidates from this work, we estimate that our knowledge of the young, massive cluster population is about half complete. We indicate locations on the Galactic plane possibly hosting the remaining unidentified massive clusters and associations.

Future astrometric, spectroscopic and/or X-ray followup observations can confirm the candidates, measure their physical properties and determine their environmental impact. With such confirmation, the candidate clusters and associations within the most luminous SFCs in the Galaxy will enable the more accurate determination of the upper end of the Galactic CMF. 
We thank B. Arsenault and H. White for assistance in the preliminary stages of this work. We also thank $\mathrm{N}$. Murray, R. Kennicutt, J. Graham, and P.G. Martin for the many helpful discussions and comments. This publication makes use of data products from the Two Micron All Sky Survey, which is a joint project of the University of Massachusetts and the Infrared Processing and Analysis Center/California Institute of Technology, funded by the National Aeronautics and Space Administration and the National Science Foundation. C.D.M and D-S.M. acknowledge support from the Natural Science and Engineering Research Council of Canada. This paper was studied with the support of the Ministry of Education Science and Technology (MEST) and the Korean Federation of Science and Technology Societies (KOFST).

\section{REFERENCES}

Anantharamaiah, K. R. 1985, Journal of Astrophysics and Astronomy, 6, 203

Andersen, J. 1991, A\&A Rev., 3, 91

Ascenso, J., Alves, J., Beletsky, Y., \& Lago, M. T. V. T. 2007, A\&A, 466, 137

Benjamin, R. A., et al. 2003, PASP, 115, 953

Bica, E., \& Bonatto, C. 2011, A\&A, 530, A32+

Bica, E., Dutra, C. M., Soares, J., \& Barbuy, B. 2003, A\&A, 404, 223

Bonatto, C., Santos, Jr., J. F. C., \& Bica, E. 2006, A\&A, 445, 567

Borissova, J., Ivanov, V. D., Minniti, D., Geisler, D., \& Stephens, A. W. 2005, A\&A, 435, 95

Brandner, W., Clark, J. S., Stolte, A., Waters, R., Negueruela, I. \& Goodwin, S. P. 2008, A\&A, 478, 137

Bressan, A., Fagotto, F., Bertelli, G., \& Chiosi, C. 1993, A\&AS, 100, 647

Cardelli, J. A., Clayton, G. C., \& Mathis, J. S. 1989, ApJ, 345, 245

Casertano, S., \& Hut, P. 1985, ApJ, 298, 80

Chandar, R., et al. 2010, ApJ, 719, 966

Chu, Y.-H. 2008, in IAU Symposium, Vol. 250, IAU Symposium, ed. F. Bresolin, P. A. Crowther, \& J. Puls, 341-354

Clark, J. S., et al. 2009, A\&A, 498, 109

Conti, P. S., \& Blum, R. D. 2002, ApJ, 564, 827

Conti, P. S., \& Crowther, P. A. 2004, MNRAS, 355, 899

Davies, B., Figer, D. F., Kudritzki, R.-P., MacKenty, J., Najarro, F., \& Herrero, A. 2007, ApJ, 671, 781

Dutra, C. M., Bica, E., Soares, J., \& Barbuy, B. 2003, A\&A, 400, 533

Fall, S. M., Chandar, R., \& Whitmore, B. C. 2009, ApJ, 704, 453

Figer, D. F., Kim, S. S., Morris, M., Serabyn, E., Rich, R. M., \& McLean, I. S. 1999, ApJ, 525, 750

Figer, D. F., MacKenty, J. W., Robberto, M., Smith, K., Najarro, F., Kudritzki, R. P., \& Herrero, A. 2006, ApJ, 643, 1166

Froebrich, D., Scholz, A., \& Raftery, C. L. 2007, MNRAS, 374, 399

Grabelsky, D. A., Cohen, R. S., Bronfman, L., \& Thaddeus, P. 1988, ApJ, 331, 181

Hanson, M. M., \& Bubnick, B. F. 2008, PASP, 120, 150

Hanson, M. M., \& Popescu, B. 2008, in IAU Symposium, Vol. 250, IAU Symposium, ed. F. Bresolin, P. A. Crowther, \& J. Puls, 307-312
Harayama, Y., Eisenhauer, F., \& Martins, F. 2008, ApJ, 675, 1319 Hunter, J. D. 2007, Computing In Science \& Engineering, 9, 90 Ivanov, V. D., Borissova, J., Bresolin, F., \& Pessev, P. 2005, A\&A, 435, 107

Ivanov, V. D., Messineo, M., Zhu, Q., Figer, D., Borissova, J., Kurtev, R., \& Ivanov, G. R. 2010, in IAU Symposium, Vol. 266, IAU Symposium, ed. R. de Grijs \& J. R. D. Lépine, 203-210

Kennicutt, Jr., R. C., Edgar, B. K., \& Hodge, P. W. 1989, ApJ, 337,761

Knödlseder, J. 2000, A\&A, 360, 539

Kroupa, P. 2001, MNRAS, 322, 231

Larsen, S. S. 2009, A\&A, 494, 539

Larsen, S. S., \& Richtler, T. 2000, A\&A, 354, 836

Lawrence, A., et al. 2007, MNRAS, 379, 1599

Lee, E. J., Murray, N., \& Rahman, M. 2012, ApJ, 752, 146

Marshall, D. J., Robin, A. C., Reylé, C., Schultheis, M., \& Picaud, S. 2006, A\&A, 453, 635

Martins, F., \& Plez, B. 2006, A\&A, 457, 637

Martins, F., Schaerer, D., \& Hillier, D. J. 2005, A\&A, 436, 1049

McKee, C. F., \& Williams, J. P. 1997, ApJ, 476, 144

Mercer, E. P., et al. 2005, ApJ, 635, 560

Minniti, D., et al. 2010, New A, 15, 433

Muench, A. A., Lada, E. A., Lada, C. J., \& Alves, J. 2002, ApJ, 573,366

Murray, N., \& Rahman, M. 2010, ApJ, 709, 424

Nishiyama, S., Nagata, T., Tamura, M., Kandori, R., Hatano, H., Sato, S., \& Sugitani, K. 2008, ApJ, 680, 1174

Nishiyama, S., Tamura, M., Hatano, H., Kato, D., Tanabé, T., Sugitani, K., \& Nagata, T. 2009, ApJ, 696, 1407

Norman, C. A., \& Ikeuchi, S. 1989, ApJ, 345, 372

Paumard, T., et al. 2006, ApJ, 643, 1011

Pickles, A. J. 1998, PASP, 110, 863

Piskunov, A. E., Kharchenko, N. V., Schilbach, E., Röser, S., Scholz, R.-D., \& Zinnecker, H. 2008, A\&A, 487, 557

Povich, M. S., et al. 2011, ApJS, 194, 6

Rahman, M., Matzner, C., \& Moon, D.-S. 2011a, ApJ, 728, L37

Rahman, M., Moon, D.-S., \& Matzner, C. D. 2011b, ApJ, 743, L28

Rahman, M., \& Murray, N. 2010, ApJ, 719, 1104

Reynolds, R. J., Sterling, N. C., \& Haffner, L. M. 2001, ApJ, 558, L101

Robin, A. C., Reylé, C., Derrière, S., \& Picaud, S. 2003, A\&A, 409, 523

Skrutskie, M. F., et al. 2006, AJ, 131, 1163

Smith, N., \& Brooks, K. J. 2007, MNRAS, 379, 1279

Smith, T. R., \& Kennicutt, Jr., R. C. 1989, PASP, 101, 649

Soares, J. B., Bica, E., Ahumada, A. V., \& Clariá, J. J. 2008, A\&A, 478, 419

Stead, J. J., \& Hoare, M. G. 2009, MNRAS, 400, 731

Stolte, A., Brandner, W., Brandl, B., Zinnecker, H., \& Grebel, E. K. 2004, AJ, 128, 765

Weaver, R., McCray, R., Castor, J., Shapiro, P., \& Moore, R. 1977, ApJ, 218, 377

Whitmore, B. C., Chandar, R., \& Fall, S. M. 2007, AJ, 133, 1067

Wolff, S. C., Strom, S. E., Dror, D., \& Venn, K. 2007, AJ, 133, 1092

Wright, N. J., Drake, J. J., Drew, J. E., \& Vink, J. S. 2010, ApJ, 713,871 
Table 1

Candidate Clusters/Associations

\begin{tabular}{|c|c|c|c|c|c|c|c|c|c|c|c|c|c|c|}
\hline SFC & $\begin{array}{c}l \\
(\mathrm{deg})\end{array}$ & $\begin{array}{c}b \\
(\mathrm{deg})\end{array}$ & $\begin{array}{c}\mathrm{D}^{\mathrm{b}} \\
(\mathrm{kpc})\end{array}$ & $\begin{array}{c}\text { smaj } \\
(\operatorname{arcmin})\end{array}$ & $\begin{array}{c}\text { smin } \\
(\operatorname{arcmin})\end{array}$ & $\begin{array}{c}P A \\
(\operatorname{deg})\end{array}$ & $j$ & $\operatorname{Min}^{A}$ & ${ }^{\prime}{ }^{\prime} \operatorname{Max}$ & $\begin{array}{c}A_{K}{ }^{\mathrm{c}} \\
\text { (Model) }\end{array}$ & $N_{T O T}$ & $N_{B G}$ & $\sigma_{B G}$ & ES \\
\hline $5 \mathrm{a}$ & 23.108 & -0.439 & $4.9,(10.8)$ & 6 & 4 & -52 & 40 & 0.5 & 0.7 & $1.0,2.4$ & 106 & 57 & 18 & 2.7 \\
\hline $5 b$ & 22.928 & -0.252 & $4.9,(10.8)$ & 3 & 2 & -24 & 40 & 0.5 & 0.7 & $1.1,1.4$ & 32 & 18 & 7 & 2.0 \\
\hline 6 & 23.389 & -0.187 & 9.2 & 9 & 7 & 63 & 29 & 0.6 & 0.7 & 1.6 & 84 & 34 & 13 & 3.8 \\
\hline $7 \mathrm{a}$ & 23.993 & 0.110 & $5.8,(9.8)$ & 5 & 3 & 0 & 37 & 0.5 & 0.6 & $1.4,2.2$ & 18 & 8 & 4 & 2.3 \\
\hline $7 \mathrm{~b}$ & 23.600 & 0.166 & $5.8,(9.8)$ & 7 & 4 & 0 & 37 & 0.5 & 0.6 & $0.7,1.7$ & 38 & 19 & 9 & 2.2 \\
\hline 8 & 24.152 & -0.374 & 10.3 & 13 & 11 & -68 & 114 & 1.7 & 2.0 & 2.3 & 392 & 315 & 137 & 0.6 \\
\hline 10 & 25.058 & 0.165 & 6.1 & 15 & 11 & 72 & 108 & 1.5 & 1.7 & 1.2 & 690 & 341 & 165 & 2.1 \\
\hline 12 & 25.995 & 0.127 & $8.8,(6.5)$ & 7 & 5 & 0 & 107 & 1.6 & 1.8 & $1.8,1.4$ & 184 & 66 & 34 & 3.4 \\
\hline 17 & 30.610 & 0.167 & 6.3 & 24 & 16 & -13 & 41 & 0.6 & 0.7 & 0.7 & 409 & 266 & 61 & 2.3 \\
\hline 19 & 34.294 & 0.220 & 2.2 & 9 & 5 & -30 & 27 & 0.6 & 0.7 & 0.6 & 48 & 24 & 10 & 2.3 \\
\hline 22 & 37.114 & -0.577 & 10.5 & 7 & 4 & -41 & 37 & 1.5 & 1.6 & 2.2 & 55 & 18 & 11 & 3.2 \\
\hline 23 & 38.294 & -0.004 & $3.5,(9.9)$ & 6 & 6 & 0 & 35 & 1.3 & 1.4 & $1.1,2.4$ & 48 & 24 & 13 & 1.9 \\
\hline 25 & 49.318 & -0.215 & 5.7 & 7 & 6 & -38 & 155 & 0.6 & 0.8 & 0.6 & 198 & 124 & 28 & 2.6 \\
\hline 26 & 283.976 & -0.868 & 4 & 19 & 10 & -79 & 61 & 0.4 & 0.5 & 0.2 & 278 & 196 & 47 & 1.7 \\
\hline $28 \mathrm{a}$ & 291.177 & -0.952 & 7.4 & 7 & 6 & 0 & 121 & 0.8 & 1.0 & 0.7 & 175 & 92 & 28 & 3.0 \\
\hline $28 \mathrm{~b}$ & 292.238 & -0.666 & 7.4 & 11 & 9 & -23 & 121 & 0.8 & 1.0 & 1.0 & 333 & 193 & 47 & 3.0 \\
\hline $29^{\mathrm{a}}$ & 298.550 & -0.720 & 9.7 & 11 & 10 & 0 & 100 & 1.0 & 1.4 & 1.0 & 897 & 491 & 102 & 4.0 \\
\hline 30 & 311.034 & 0.454 & 3.5 & 7 & 4 & 67 & 63 & 0.4 & 0.5 & 0.9 & 43 & 34 & 10 & 0.9 \\
\hline 31 & 311.539 & 0.347 & 7.4 & 8 & 7 & -63 & 48 & 0.3 & 0.4 & 1.2 & 87 & 45 & 13 & 3.4 \\
\hline 33 & 328.013 & -0.568 & 3.7 & 23 & 7 & -7 & 193 & 0.4 & 0.6 & 0.3 & 735 & 502 & 138 & 1.7 \\
\hline $34 \mathrm{a}$ & 333.514 & -0.080 & 3.4 & 6 & 5 & 0 & 604 & 0.3 & 0.7 & 0.2 & 473 & 299 & 70 & 2.5 \\
\hline $34 b$ & 332.931 & -0.569 & 3.4 & 17 & 7 & -23 & 604 & 0.3 & 0.7 & 0.3 & 1766 & 1239 & 212 & 2.5 \\
\hline 36 & 336.537 & -0.162 & $5.4,(10.2)$ & 6 & 4 & -50 & 26 & 1.0 & 1.1 & $0.8,2.0$ & 36 & 12 & 6 & 4.1 \\
\hline 37 & 337.243 & -0.011 & 10.9 & 10 & 6 & 9 & 495 & 1.3 & 1.8 & 1.6 & 781 & 460 & 91 & 3.5 \\
\hline 38 & 337.787 & -0.382 & 3.5 & 9 & 7 & -60 & 44 & 0.4 & 0.5 & 0.9 & 91 & 50 & 16 & 2.6 \\
\hline 39 & 338.361 & 0.197 & $13.3,(2.5)$ & 7 & 7 & 90 & 14 & 1.8 & 1.9 & $1.8,0.2$ & 24 & 11 & 5 & 2.4 \\
\hline
\end{tabular}

a Candidate cluster properties from Rahman et al. (2011a)

c Where a kinematic distance ambiguity exists, we list the distance most consistent with candidate extinction first, and the less consistent distance in parentheses.

c Model extinction from Marshall et al. (2006) 


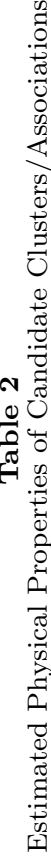

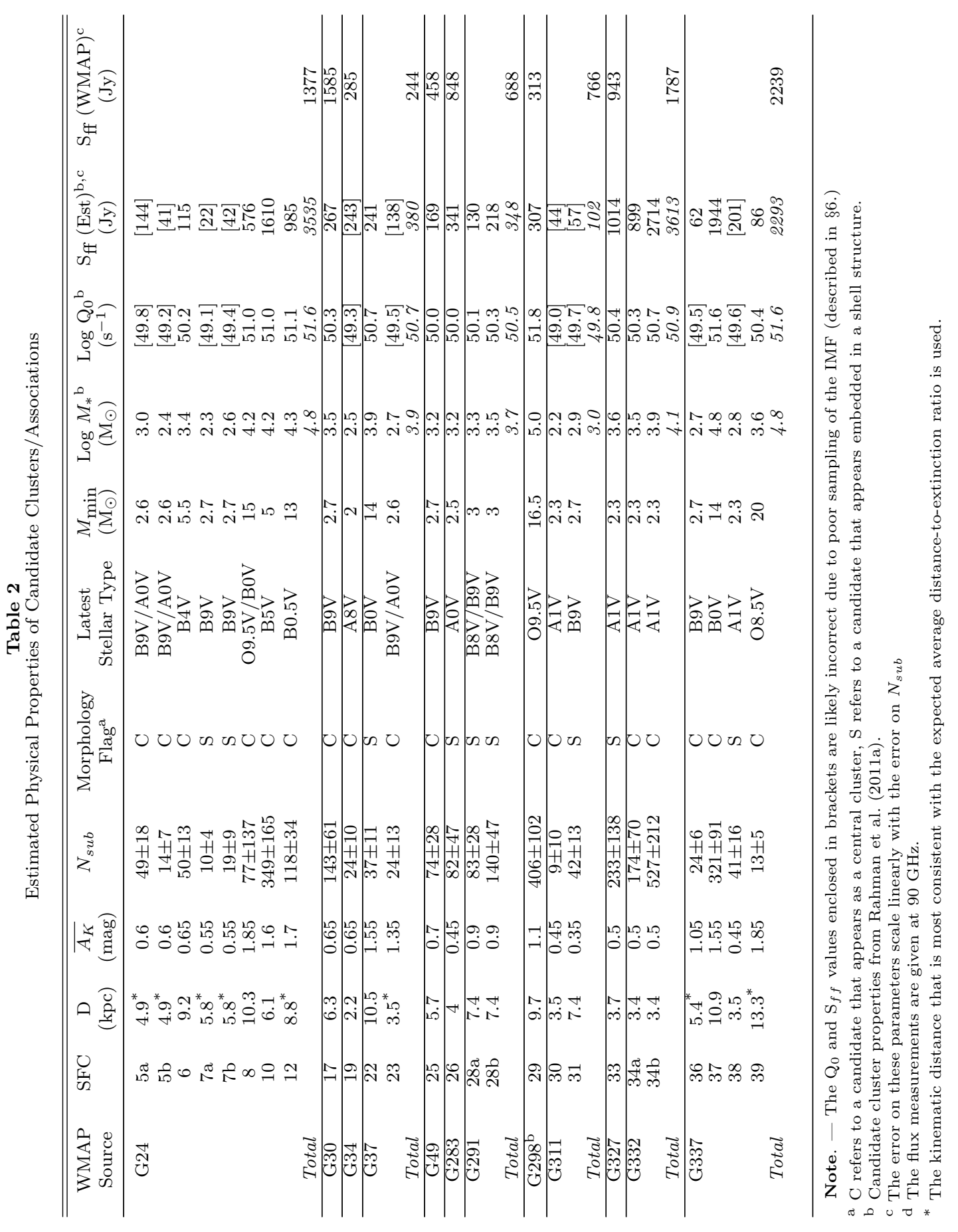


Table 3

Census of Massive $\left(\gtrsim 10^{4} \mathrm{M}_{\odot}\right)$, Young $(\lesssim 4 \mathrm{Myr})$ Clusters \& OB Associations in the Galaxy

\begin{tabular}{ccccc}
\hline \hline Galactic Region & Mass $\left(\log \mathrm{M}_{\odot}\right)$ & Distance $(\mathrm{kpc})$ & Name & Reference \\
\hline Galactic Centre & 4.1 & 8.5 & Central Cluster & $(5)$ \\
& 4.0 & 8.5 & Arches & $(3)$ \\
& 4.0 & 8.5 & Quintuplet & $(3)$ \\
\hline G24 & $\geq 4.2^{*}$ & 10.3 & SFC 8 & $(1)$ \\
& $\geq 4.2^{*}$ & 6.1 & SFC 10 & $(1)$ \\
& $\geq 4.3^{*}$ & $8.8^{*}$ & SFC 12 & $(1)$ \\
\hline G37 & $\geq 3.9^{*}$ & 10.5 & SFC 22 & $(1)$ \\
\hline G43 & $\sim 4$ & 11.4 & W49A & $(2)$ \\
\hline G80 & 4.4 & 1.5 & Cygnus OB2 & $(8)$ \\
\hline G283 & $>4.0$ & 2.8 & RCW49, Westerlund2 & $(9)$ \\
\hline G287 & $>4.3$ & 2.3 & Carina & $(7)$ \\
\hline G298 & 4.2 & 6.0 & NGC 3603 & $(4)$ \\
\hline G332 & 5.0 & 9.7 & Dragonfish & $(6)$ \\
\hline G337 & $\geq 4.1^{*}$ & 3.2 & SFC 34a,b & $(1)$ \\
\hline & $\geq 4.8^{*}$ & 10.9 & SFC 37 & $(1)$
\end{tabular}

References. - (1) This work; (2) Conti \& Blum (2002); (3) Figer et al. (1999); (4) Harayama et al. (2008); (5) Paumard et al. (2006); (6) Rahman et al. (2011a); (7) Smith \& Brooks (2007); (8) Wright et al. (2010); (9) Ascenso et al. (2007);

Note. - Wolff et al. (2007) use an IMF-sensitive extrapolation over a small mass range to determine the masses of IC 1805 and NGC 6611. The extrapolated mass of NGC 6611 is $10^{4.4}$ $\mathrm{M}_{\odot}$, in contrast to more recent work by Bonatto et al. (2006) that revise the minimum cluster mass down to $10^{3.2} \mathrm{M}_{\odot}$. In both of these cases, the free-free luminosities of the regions from Murray \& Rahman (2010) are inconsistent with young clusters with $\mathrm{M}>10^{4} \mathrm{M}_{\odot}$, indicating smaller masses. Consequently, we exclude both of these regions from this census.

* Candidate Cluster/Association from this work.

\# Unresolved kinematic distance ambiguity. The distance indicated is the most consistent with the model extinction to the candidate. 
